\title{
Synthesis of C1-C13 Fragment of Eribulin on a Kilogram Scale
}

Seong Taek Kim, Yongseo Park, Namhyeon Kim, Jaeun Gu, Wongyoung Son, Jisu Hur, KangHee Lee, Areum Baek, Ju Young Song, U Bin Kim, Kee-Young Lee, Chang-Young Oh, Seokhwi Park, Hyunik Shin*

R\&D Center, Yonsung Fine Chemicals Co., Ltd., Innoplex-2-dong, 306, Sinwon-ro, Yeongtong-gu, Suwon-si, Gyeonggi-do, 16675, Republic of Korea

hishin@yonsungchem.co.kr

\section{Table of Contents}

Experimental procedures for synthesis of C1-C13 fragment (3) S2-S6

Copies of ${ }^{1} \mathrm{H}$ and ${ }^{13} \mathrm{C}$ NMR spectra

S7-S21 


\section{Detailed experimental procedure for synthesis of 9}

Ethyl $\quad(E)-3-((2 R, 3 S)-3-((2 S, 3 R)-3-((S)$-hydroxy $((R)-1,4-d i o x a s p i r o[4.5]$ decan-2-yl)methyl)-1,4dioxaspiro[4.5]decan-2-yl)oxiran-2-yl)acrylate (9)

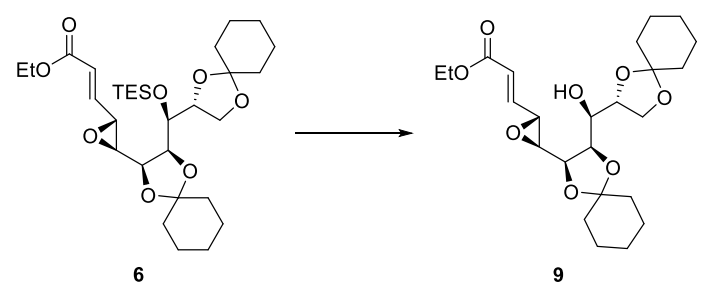

To a solution of $6(6.04 \mathrm{~kg}, 10.7 \mathrm{~mol})$ in THF $(60 \mathrm{~L})$ was added imidazole $\cdot \mathrm{HCl}(0.78 \mathrm{~kg}, 7.46 \mathrm{~mol}$, 0.7 equiv) and the reaction mixture was cooled to $5{ }^{\circ} \mathrm{C}$. TBAF $(3.9 \mathrm{~kg}, 14.9 \mathrm{~mol}, 1.0 \mathrm{M}$ in THF, 1.4 equiv) was then dropwise added and the reaction mixture was warmed to room temperature, and the reaction mixture was stirred for $2 \mathrm{~h}$. [In-process check; TLC $(\mathrm{Hex} / \mathrm{EtOAc}=4: 1)$ ]. Upon completion of the reaction, the reaction mixture was chilled to $5{ }^{\circ} \mathrm{C}$ and $\mathrm{H}_{2} \mathrm{O}(30 \mathrm{~L})$ was added followed by EtOAc $(30 \mathrm{~L})$ and the mixture was stirred for further $30 \mathrm{~min}$. Layers were than separated, and aqueous layer was extracted with EtOAc. The combined organic fractions were washed with $\mathrm{H}_{2} \mathrm{O}, 3$ times to completely remove the residual fluoride ion. Presence of halides was tested by taking an aliquot of the aqueous washings, adding $\mathrm{AgNO}_{3}$ and observing for precipitation. If there was precipitation observed, the organic layer was washed again with $\mathrm{H}_{2} \mathrm{O}$.

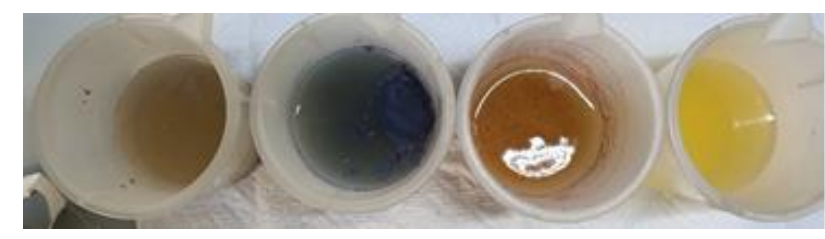

Aq. layer

$$
\begin{array}{ccc}
1^{\text {st }} \text { wash, } & 2^{\text {nd }} \text { wash } & 3^{\text {rd }} \text { wash (no precipitate) } \\
\mathrm{AgNO}_{3} \text { added } & \mathrm{AgNO}_{3} \text { added } & \mathrm{AgNO}_{3} \text { added }
\end{array}
$$

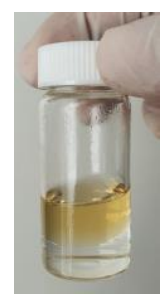

after $3^{\text {rd }}$ wash (no precipitation)

Upon confirmation of no presence of residual halide ions derived from TBAF and imidazole $\cdot \mathrm{HCl}$ in the aqueous wash, combined organic extracts were dried $\left(\mathrm{Na}_{2} \mathrm{SO}_{4}\right)$ and concentrated in vacuo to afford the desired crude product 9 as a yellow oil $(3.73 \mathrm{~kg}, 77 \%) .{ }^{1} \mathrm{H}$ NMR $\left(300 \mathrm{MHz}, \mathrm{CDCl}_{3}\right): \delta 6.68$ (dd, $J$ $=15.7,7.1 \mathrm{~Hz}, 1 \mathrm{H}), 6.17(\mathrm{dd}, J=15.7,0.6 \mathrm{~Hz}, 1 \mathrm{H}), 4.29-4.06(\mathrm{~m}, 5 \mathrm{H}), 3.94-3.78(\mathrm{~m}, 3 \mathrm{H}), 3.43(\mathrm{dd}$, $J=7.0,1.4 \mathrm{~Hz}, 1 \mathrm{H}), 3.27(\mathrm{dd}, J=7.8,1.9 \mathrm{~Hz}, 1 \mathrm{H}), 2.49(\mathrm{~d}, J=7.6 \mathrm{~Hz}, 1 \mathrm{H}), 1.73-1.24(\mathrm{~m}, 23 \mathrm{H}) ;{ }^{13} \mathrm{C}$ NMR (75 MHz, $\left.\mathrm{CDCl}_{3}\right): \delta 165.49,143.09,124.64,110.38,110.28,77.22,76.13,69.12,65.58,60.67$, $58.50,55.80,36.93,36.08,34.86,34.38,25.14,25.03,24.00,23.82,23.65,14.19,1.74,-0.01$.

\section{Experimental procedures for synthesis of C1-C13 fragment from 13 to 3}

Ethyl 2-((3a'R,4'S,5a'S,8'R,9a'S,9b'S)-4'-((R)-1,4-dioxaspiro[4.5]decan-2-yl)octahydrospiro[cyclohexane-1,2'$[1,3]$ dioxolo[4,5- $d]$ pyrano[3,2-b]pyran]-8'-yl)acetate (SI1) 


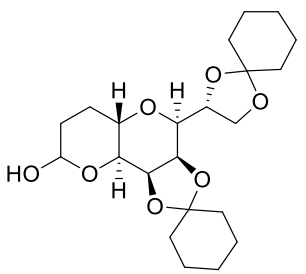

13

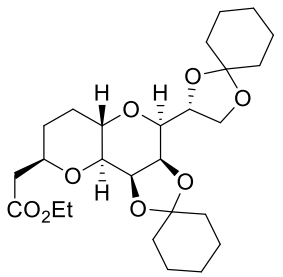

SI1

To a chilled solution of triehtyl phophonoacetate $\left(3 \mathrm{~kg}, 13.37 \mathrm{~mol}, 2.3\right.$ equiv) in THF ( $38 \mathrm{~L}$ ) at $0{ }^{\circ} \mathrm{C}$ was added $\mathrm{KO}^{t} \mathrm{Bu}(1.5 \mathrm{~kg}, 13.37 \mathrm{~mol}, 2.3$ equiv) and the reaction mixture was stirred for $1 \mathrm{~h}$. A solution of $\mathbf{1 3}(4.2 \mathrm{~kg}, 5.85$ mol) in THF (4.2 L) was then dropwise added. Reaction temperature was elevated to $60{ }^{\circ} \mathrm{C}$ and the reaction mixture was stirred for $4 \mathrm{~h}$. Upon completion of the reaction temperature was cooled to room temperature, and $\mathrm{NH}_{4} \mathrm{Cl}(21 \mathrm{~L}$, aq., $10 \%)$ and EtOAc $(21 \mathrm{~L})$ were added to the reaction mixture and stirred for $30 \mathrm{~min}$. Layers were than separated, and organic layer was collected. The aqueous layer was extracted with EtOAc $(8.4 \mathrm{~L})$. The combined organic fractions were dried $\left(\mathrm{Na}_{2} \mathrm{SO}_{4}\right)$ and concentrated in vacuo and purified by flash chromatography (Hexane/EtOAc $=3: 1)$ to afford desired product SI1 $(3.6 \mathrm{~kg}, 76 \%) .{ }^{1} \mathrm{H}$ NMR $(300 \mathrm{MHz}$, $\left.\mathrm{CDCl}_{3}\right): \delta 4.52(\mathrm{dd}, J=8.3,2.9 \mathrm{~Hz}, 1 \mathrm{H}), 4.34-4.22(\mathrm{~m}, 2 \mathrm{H}), 4.17-4.06(\mathrm{~m}, 3 \mathrm{H}), 3.95-3.76(\mathrm{~m}, 3 \mathrm{H}), 3.72(\mathrm{dd}, J$ $=7.1,1.5 \mathrm{~Hz}, 1 \mathrm{H}), 3.49(\mathrm{dd}, J=10.2,3.0 \mathrm{~Hz}, 1 \mathrm{H}), 2.70(\mathrm{dd}, J=16.0,6.8 \mathrm{~Hz}, 1 \mathrm{H}), 2.41(\mathrm{dd}, J=16.0,6.2 \mathrm{~Hz}$, $1 \mathrm{H}), 2.20-2.13(\mathrm{~m}, 1 \mathrm{H}), 1.84-1.22(\mathrm{~m}, 26 \mathrm{H}) ;{ }^{13} \mathrm{C} \mathrm{NMR}\left(75 \mathrm{MHz}, \mathrm{CDCl}_{3}\right): \delta 171.04,111.07,110.09,76.30$, $75.21,74.18,71.82,71.37,66.12,65.51,60.45,40.53,36.32,35.63,35.25,33.51,30.00,25.14,23.99,23.77$, $23.65,14.17$.

Ethyl 2-((3a'R,4'S,5a'S,8'R,9a'S,9b'S)-4'-((R)-1,2-dihydroxyethyl)octahydrospiro[cyclohexane-1,2'$[1,3]$ dioxolo[4,5- $d]$ pyrano[3,2-b]pyran]-8'-yl)acetate (SI2)

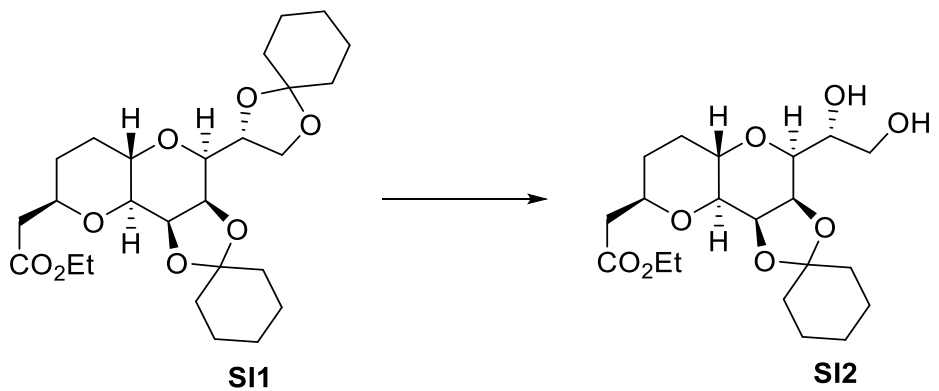

A solution of SI1 (3.36 kg, $7.48 \mathrm{~mol})$ in $\mathrm{AcOH}(57.6 \mathrm{~L})$ and water $(14.4 \mathrm{~L})$ was heated to $40{ }^{\circ} \mathrm{C}$ and the reaction mixture was stirred for $6 \mathrm{~h}$. The reaction mixture was concentrated in vauco then to the residue was added toluene $(36 \mathrm{~L})$ and the reaction mixture was again concentrated in vacuo. Azeotropic concentration with toluene was then repeated one more time in vacuo (toluene, $36 \mathrm{~L}) . \mathrm{CH}_{2} \mathrm{Cl}_{2}(30 \mathrm{~L})$ was added followed by $\mathrm{NaHCO}_{3}(54 \mathrm{~L}, 10 \%$ aq.) to adjust the $\mathrm{pH}$ to 7 . The layers were than separated and organic layer was collected. The aqueous layer was extracted with $\mathrm{CH}_{2} \mathrm{Cl}_{2}(15 \mathrm{~L})$ and combined organic extracts were dried $\left(\mathrm{Na}_{2} \mathrm{SO}_{4}\right)$, concentrated in vacuo, and purified by flash chromatography $(\mathrm{Hex} / \mathrm{EtOAc}=3: 1)$ to afford desired product SI2 $(2.2 \mathrm{~kg}, 73 \%) .{ }^{1} \mathrm{H}$ NMR (300 $\left.\mathrm{MHz}, \mathrm{CDCl}_{3}\right): \delta 4.56(\mathrm{dd}, J=8.4,2.9 \mathrm{~Hz}, 1 \mathrm{H}), 4.45(\mathrm{dd}, J=8.4,1.4 \mathrm{~Hz}, 1 \mathrm{H}), 4.13(\mathrm{q}, J=7.1 \mathrm{~Hz}, 2 \mathrm{H}), 3.95-3.82$ $(\mathrm{m}, 3 \mathrm{H}), 3.81-3.75(\mathrm{~m}, 3 \mathrm{H}), 3.51(\mathrm{dd}, J=10.2,2.9 \mathrm{~Hz}, 1 \mathrm{H}), 3.15(\mathrm{br} \mathrm{s}, 1 \mathrm{H}), 2.70(\mathrm{dd}, J=16.0,6.8 \mathrm{~Hz}, 1 \mathrm{H}), 2.45-$ $2.38(\mathrm{~m}, 2 \mathrm{H}), 2.14-2.08(\mathrm{~m}, 1 \mathrm{H}), 1.84-1.22(\mathrm{~m}, 16 \mathrm{H}) ;{ }^{13} \mathrm{C}$ NMR $\left(75 \mathrm{MHz}, \mathrm{CDCl}_{3}\right): \delta 171.00,111.19,76.22$, $75.30,74.52,72.05,71.34,69.84,66.33,62.57,60.51,40.50,35.55,33.29,30.55,29.94,25.06,24.04,23.62$, 14.19.

Ethyl 2-((3a'S,4'R,5a'S,8'R,9a'S,9b'S)-4'-formyloctahydrospiro[cyclohexane-1,2'-[1,3]dioxolo[4,5-d]pyrano[3,2$b]$ pyran]-8'-yl)acetate (SI3) 


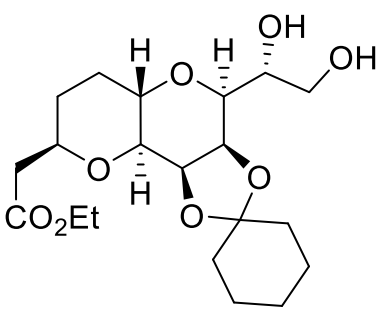

$\mathrm{S} 12$

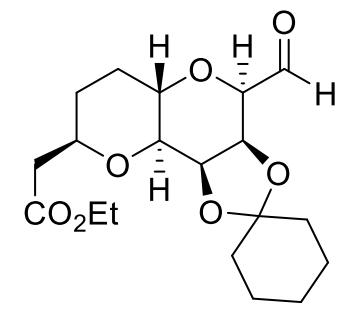

$\mathrm{S} \mid 3$

To a chilled solution of SI2 $(2.2 \mathrm{~kg}, 4.02 \mathrm{~mol})$ in EtOAc $(8.8 \mathrm{~L})$ and $\mathrm{H}_{2} \mathrm{O}(8.8 \mathrm{~L})$ at $10{ }^{\circ} \mathrm{C}$ was added $\mathrm{NaIO}_{4}(0.9$ $\mathrm{kg}, 4.22 \mathrm{~mol}, 1.05$ equiv) portion wise and the reaction mixture was stirred for $2 \mathrm{~h}$. Upon completion of the reaction, $\mathrm{NaCl}(2.2 \mathrm{~kg}, 1 \mathrm{wt} \%)$ was added and the reaction mixture was stirred for further $30 \mathrm{~min}$. The solids were then filtered off and layers were separated and organic layer was collected. The aqueous layer was extracted with EtOAc and combined organic layers were washed with $20 \%$ solution of $\mathrm{NaCl}(8.8 \mathrm{~L}, 20 \%$, aq.). The combined organic extracts were dried $\left(\mathrm{Na}_{2} \mathrm{SO}_{4}\right)$ and concentrated in vacuo to afford desired product $\mathbf{S I 3}\left(1.9 \mathrm{~kg}\right.$, quant.). ${ }^{1} \mathrm{H}$ $\operatorname{NMR}\left(300 \mathrm{MHz}, \mathrm{CDCl}_{3}\right): \delta 9.62(\mathrm{~d}, J=0.6 \mathrm{~Hz}, 1 \mathrm{H}), 4.70(\mathrm{dd}, J=8.2,2.2 \mathrm{~Hz}, 1 \mathrm{H}), 4.58(\mathrm{dd}, J=8.2,3.0 \mathrm{~Hz}, 1 \mathrm{H})$, 4.17-4.10 (m, 3H), 4.05-3.96 (m, 1H), 3.90-3.82 (m, 1H), $3.43(\mathrm{dd}, J=7.1 \mathrm{~Hz}, 2 \mathrm{H}), 3.90-3.82(\mathrm{~m}, 1 \mathrm{H}), 3.43$ $(\mathrm{dd}, J=10.2,3.0 \mathrm{~Hz}, 1 \mathrm{H}), 2.71(\mathrm{dd}, J=16.1,6.9 \mathrm{~Hz}, 1 \mathrm{H}), 2.42(\mathrm{dd}, J=16.1,6.1 \mathrm{~Hz}, 1 \mathrm{H}), 2.25-2.18(\mathrm{~m}, 1 \mathrm{H})$, $1.83-1.23(\mathrm{~m}, 13 \mathrm{H}) ;{ }^{13} \mathrm{C}$ NMR $\left(75 \mathrm{MHz}, \mathrm{CDCl}_{3}\right): \delta 200.87,170.98,111.46,76.53,75.34,75.23,75.05,70.93$, $66.08,60.54,40.44,35.53,33.29,30.37,29.84,25.07,23.84,23.44,14.19$.

Ethyl 2-((3a'R,4'S,5a'S,8' $\left.R, 9 \mathrm{a}^{\prime} S, 9 \mathrm{~b}^{\prime} S\right)-4^{\prime}-((S, E)-1$-hydroxy-3-(trimethylsilyl)allyl)octahydrospiro[cyclohexane$1,2^{\prime}-[1,3]$ dioxolo[4,5-d]pyrano[3,2-b]pyran]-8'-yl)acetate (SI4)<smiles>CCOC(=O)C[C@H]1CC[C@@H]2O[C@H](C=O)[C@H]3OC4(CCCCC4)O[C@H]3[C@H]2O1</smiles>

SI3

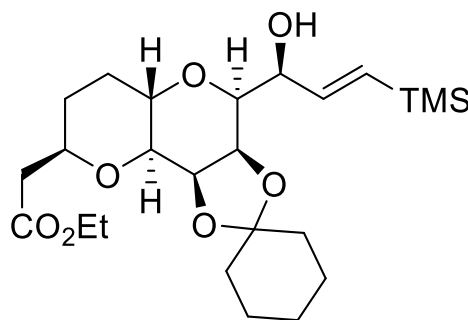

SI4

A degassed solution of MeO-(S)-CBS-Ligand (29.8 g, 96 mmol, 0.11 equiv) and $\mathrm{CrCl}_{2}$ (10.68 g, 87 mmol, 0.10 equiv) in $\mathrm{MeCN}(2.24 \mathrm{~L})$ was stirred for $1 \mathrm{~h}$. Reaction temperature was cooled to $15{ }^{\circ} \mathrm{C}$ and $\mathrm{LiCl}(73.6 \mathrm{~g}, 1.74$ mol, 2 equiv), Mn powder (95.44 g, $1.74 \mathrm{~mol}, 2$ equiv), and $\mathrm{NiCl}_{2} \cdot \mathrm{DMP}$ complex (3.82 $\mathrm{g}, 17 \mathrm{mmol}, 0.02$ equiv) were added. A solution of SI3 $(320 \mathrm{~g}, 869 \mathrm{mmol})$ in $\mathrm{MeCN}(0.64 \mathrm{~L})$ was then added followed by addition of (E)-(2-bromovinyl)trimethylsilane (199.98 mL, $1.3 \mathrm{~mol}, 1.5$ equiv) and $\mathrm{ZrCp}_{2} \mathrm{Cl}_{2}$ (253.9 g, $869 \mathrm{mmol}, 1$ equiv) and the reaction mixture was stirred for $30 \mathrm{~min}$ then warmed to room temperature and the reaction mixture was stirred for $19 \mathrm{~h}$. EtOAc (3.2 L) and Florisil ${ }^{\circledR}(320 \mathrm{~g})$ were added and the reaction mixture was stirred for $30 \mathrm{~min}$. The reaction mixture was filtered through a silica pad and concentrated in vacuo. The whole reaction procedure was repeated with 5 batches and combined residue was purified by flash chromatography $(\mathrm{Hex} / \mathrm{EtOAc}=5: 1)$ to afford desired product SI4 (1.18 kg, 58\%). ${ }^{1} \mathrm{H}$ NMR $\left(300 \mathrm{MHz}, \mathrm{CDCl}_{3}\right): \delta 6.19-6.06(\mathrm{~m}, 2 \mathrm{H}), 4.55-4.47(\mathrm{~m}$, $2 \mathrm{H}), 4.38-4.32(\mathrm{~m}, 1 \mathrm{H}), 4.14(\mathrm{q}, J=7.1 \mathrm{~Hz}, 2 \mathrm{H}), 3.98-3.80(\mathrm{~m}, 2 \mathrm{H}), 3.63(\mathrm{dd}, J=5.2,1.1 \mathrm{~Hz}, 1 \mathrm{H}), 3.49(\mathrm{dd}, J$ $=1.02,2.7 \mathrm{~Hz}, 1 \mathrm{H}), 3.02(\mathrm{~d}, J=7.7 \mathrm{~Hz}, 1 \mathrm{H}), 2.70(\mathrm{dd}, J=16.0,6.8 \mathrm{~Hz}, 1 \mathrm{H}), 2.41(\mathrm{dd}, J=16.0,6.2 \mathrm{~Hz}, 1 \mathrm{H})$, 2.18-2.08 (m, 1H), 1.81-1.17 (m, 16H), $0.08(\mathrm{~s}, 9 \mathrm{H}) ;{ }^{13} \mathrm{C} \mathrm{NMR}\left(75 \mathrm{MHz}, \mathrm{CDCl}_{3}\right): \delta 172.38,146.08,132.04$, $112.52,78.54,77.63,76.61,75.83,75.53,72.66,72.60,67.72,61.82,41.86,36.84,34.54,31.90,31.27,26.43$, $25.45,24.95,15.52,2.34,0.00$. 
Ethyl 2-((2R,4aS,6S,7S,8R,8a R)-7,8-dihydroxy-6-((S,E)-1-hydroxy-3-(trimethylsilyl)allyl)octahydropyrano[3,2$b]$ pyran-2-yl)acetate (SI5)<smiles>CCOC(=O)C[C@H]1CC[C@H]2O[C@H]([C@H](O)/C=C/[Y5](C)(=O)=O)[C@H]3OC4(CCCCC4)O[C@H]3[C@H]2O1</smiles>

SI4<smiles>CCOC(=O)C[C@H]1CC[C@@H]2O[C@H]([C@H](O)/C=C/[Y5](C)(=O)=O)[C@@H](O)[C@H](O)[C@]2(C)O1</smiles>

SI5

A solution of $\mathbf{S I} 4(800 \mathrm{~g}, 1.7 \mathrm{~mol})$ in $\mathrm{AcOH}(3.36 \mathrm{~kg})$ and $\mathrm{H}_{2} \mathrm{O}(3.36 \mathrm{~kg})$ was heated to $95{ }^{\circ} \mathrm{C}$ and the reaction mixture was stirred for $3 \mathrm{~h}$. The reaction mixture was cooled to $15^{\circ} \mathrm{C}$ and washed with heptane $(2.16 \mathrm{~kg} \times 2)$. Aqueous layer was collected and while keeping the reaction temperature below $15^{\circ} \mathrm{C}$, and a solution of $\mathrm{KHCO}_{3}$ $(29.9 \mathrm{~kg}, 20 \%$, aq.) and MTBE (4.76 kg) were added and stirred. Layers were separated and organic layer was collected then the organic layer was washed with $\mathrm{KHCO}_{3}(3.2 \mathrm{~L}, 5 \%$, aq.) and $\mathrm{NaCl}(3.2 \mathrm{~L}, 5 \%$, aq.). The organic layer was concentrated in vacuo then MTBE $(1.19 \mathrm{~kg})$ was added, heated to $55^{\circ} \mathrm{C}$, and stirred until residue fully dissolved. Heptane $(800 \mathrm{~g})$ was added and stirred at $45^{\circ} \mathrm{C}$. Heptane $(3.58 \mathrm{~kg})$ was further dropwise added and cooled to $10^{\circ} \mathrm{C}$ and the mixture was stirred for $15 \mathrm{~h}$. The desired product precipitated out, which was filtered and washed with heptane to afford SI5 (402 g, 60\%). ${ }^{1} \mathrm{H}$ NMR $\left(300 \mathrm{MHz}, \mathrm{CDCl}_{3}\right): \delta 6.14$ (dd, $\left.J=18.7,5.9 \mathrm{~Hz}, 1 \mathrm{H}\right)$, $5.97(\mathrm{dd}, J=18.7,0.9 \mathrm{~Hz}, 1 \mathrm{H}), 5.10-5.04(\mathrm{~m}, 1 \mathrm{H}), 4.27-4.11(\mathrm{~m}, 4 \mathrm{H}), 4.03-3.98(\mathrm{~m}, 1 \mathrm{H}), 3.91-3.87(\mathrm{~m}, 1 \mathrm{H})$, $3.76(\mathrm{dd}, J=8.8,6.2 \mathrm{~Hz}, 1 \mathrm{H}), 3.57-3.54(\mathrm{~m}, 1 \mathrm{H}), 3.22(\mathrm{~d}, J=3.2 \mathrm{~Hz}, 1 \mathrm{H}), 2.94(\mathrm{br} \mathrm{s}, 1 \mathrm{H}), 2.59$ (dd, $J=15.4,7.4$ $\mathrm{Hz}, 1 \mathrm{H}), 2.42$ (dd, $J=15.4,5.5 \mathrm{~Hz}, 1 \mathrm{H}), 1.93-1.90(\mathrm{~m}, 1 \mathrm{H}), 1.82-1.80(\mathrm{~m}, 1 \mathrm{H}), 1.46-1.39(\mathrm{~m}, 2 \mathrm{H}), 1.26$ (t, $J=$ $7.1 \mathrm{~Hz}, 4 \mathrm{H}), 0.07$ (s, 9H); ${ }^{13} \mathrm{C}$ NMR $\left(75 \mathrm{MHz}, \mathrm{CDCl}_{3}\right): \delta 170.40,146.79,133.16,79.82,76.81,76.11,75.93$, $71.86,71.46,65.47,62.03,42.12,31.74,30.37,15.55,0.00$.

Ethyl 2-((2R,4aS,6S,7R,8S,8aS)-7,8-bis((tert-butyldimethylsilyl)oxy)-6-((S,E)-1-((tert-butyldimethylsilyl)oxy)3-(trimethylsilyl)allyl)octahydropyrano[3,2-b]pyran-2-yl)acetate (SI6)<smiles>C/C=C/C(O)[C@H]1O[C@@H]2CCC(COCC)O[C@@H]2C(O)[C@H]1O</smiles>

SI5

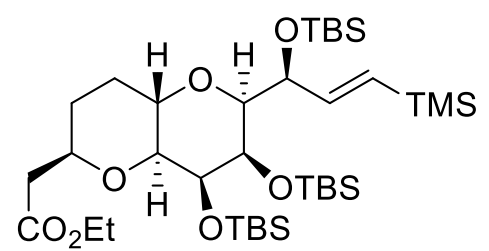

SI6

To a chilled solution of SI5 $(610 \mathrm{~g}, 1.57 \mathrm{~mol})$ in MTBE $(6.1 \mathrm{~L})$ and 2,6-lutidine (1.26 kg, $11.8 \mathrm{~mol}, 7.5$ equiv) at $0{ }^{\circ} \mathrm{C}$ was dropwise added TBSOTf $(1.45 \mathrm{~kg}, 5.49 \mathrm{~mol}, 3.5$ equiv), and the reaction mixture was stirred for 30 $\mathrm{min}$. The reaction temperature was then elevated to room temperature and stirred for $19 \mathrm{~h}$. $\mathrm{MeOH}(100 \mathrm{~mL})$ and $\mathrm{H}_{2} \mathrm{O}(9 \mathrm{~L})$ was then added and layers were separated. Organic layer was collected and washed with $1 \mathrm{~N} \mathrm{HCl}(9.9$ L) and $\mathrm{NaCl}\left(9.9 \mathrm{~L}, 5 \%\right.$, aq.) twice. Organic layer was dried $\left(\mathrm{Na}_{2} \mathrm{SO}_{4}\right)$ and concentrated and the residue was purified by flash chromatography $(\mathrm{Hex} / \mathrm{EtOAc}=50: 1)$ to afford SI6 $(1.06 \mathrm{~kg}, 92 \%) .{ }^{1} \mathrm{H}$ NMR $\left(300 \mathrm{MHz}, \mathrm{CDCl}_{3}\right)$ : $\delta 6.13(\mathrm{dd}, J=18.9,8.0 \mathrm{~Hz}, 1 \mathrm{H}), 5.81(\mathrm{dd}, J=18.9,0.5 \mathrm{~Hz}, 1 \mathrm{H}), 4.96-4.92(\mathrm{~m}, 1 \mathrm{H}), 4.11-4.02(\mathrm{~m}, 3 \mathrm{H}), 3.84-$ $3.69(\mathrm{~m}, 3 \mathrm{H}), 3.47-3.39(\mathrm{~m}, 1 \mathrm{H}), 3.85(\mathrm{dd}, J=9.6,2.3 \mathrm{~Hz}, 1 \mathrm{H}), 2.45(\mathrm{dd}, J=15.3,8.0 \mathrm{~Hz}, 1 \mathrm{H}), 2.27$ (dd, $J=$ $15.3,5.3 \mathrm{~Hz}, 1 \mathrm{H}), 1.71-1.64$ (m, 2H), 1.21-1.16 (m, 5H), 0.90 (s, 9H), 0.86 (s, 9H), 0.78 (s, 9H), 0.05--0.09 (s, $27 \mathrm{H}) ;{ }^{13} \mathrm{C}$ NMR $\left(75 \mathrm{MHz}, \mathrm{CDCl}_{3}\right): \delta 171.41,147.00,131.36,81.47,79.09,75.21,74.14,73.11,71.05,63.59$, $60.62,41.07,30.60,28.79,26.97,26.47,26.42,19.52,18.87,18.28,14.31,-1.31,-2.65,-3.07,-3.83,-4.08,-$ $4.44,-4.51$. 
Ethyl 2-((2R,4aS,6S,7R,8S,8aS)-7,8-bis((tert-butyldimethylsilyl)oxy)-6-((S,E)-1-((tert-butyldimethylsilyl)oxy)3-iodoallyl)octahydropyrano[3,2-b]pyran-2-yl)acetate (SI7)

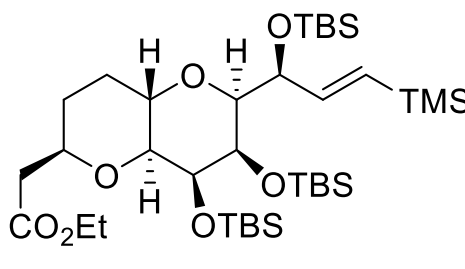

SI6

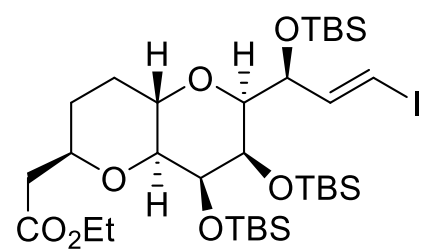

SI7

To a solution of SI6 $(1.15 \mathrm{~kg}, 1.57 \mathrm{~mol})$ in toluene $(3.45 \mathrm{~L})$ and $\mathrm{MeCN}(0.69 \mathrm{~L})$ was added TBSCl $(23.7 \mathrm{~g}$, $0.157 \mathrm{~mol}, 0.1$ equiv) followed by NIS $(1.415 \mathrm{~kg}, 6.29 \mathrm{~mol}, 4$ equiv) and the reaction mixture was stirred for 15 h. A solution of $\mathrm{Na}_{2} \mathrm{~S}_{2} \mathrm{O}_{3} / \mathrm{KHCO}_{3} /$ water $=15: 2.9: 29(23 \mathrm{~kg})$ was then dropwise added while keeping the reaction temperature below $30^{\circ} \mathrm{C}$ and the reaction mixture was further stirred for $30 \mathrm{~min}$. Layers were separated and organic layer was collected. The organic layer was washed with solution of $\mathrm{NaCl}(6.35 \mathrm{~kg}, 9 \%$, aq.) twice. Organic layer was dried $\left(\mathrm{Na}_{2} \mathrm{SO}_{4}\right)$, concentrated in vacuo and purified by flash chromatography $(\mathrm{Hex} / \mathrm{EtOAc}=$ $20: 1)$ to afford $\mathbf{S I} 7(1.12 \mathrm{~kg}, 90 \%) .{ }^{1} \mathrm{H}$ NMR $\left(300 \mathrm{MHz}, \mathrm{CDCl}_{3}\right): \delta 6.86(\mathrm{dd}, J=14.6,7.8 \mathrm{~Hz}, 1 \mathrm{H}), 6.28(\mathrm{dd}, J=$ 14.6, $0.7 \mathrm{~Hz}, 1 \mathrm{H}), 4.92-4.88(\mathrm{~m}, 1 \mathrm{H}), 4.21-4.07(\mathrm{~m}, 3 \mathrm{H}), 3.90-3.77(\mathrm{~m}, 3 \mathrm{H}), 3.51-3.42(\mathrm{~m}, 1 \mathrm{H}), 2.93(\mathrm{dd}, J=$ 9.6, $2.2 \mathrm{~Hz}, 1 \mathrm{H}), 2.53(\mathrm{dd}, J=15.4,8.1 \mathrm{~Hz}, 1 \mathrm{H}), 2.36(\mathrm{dd}, J=15.4,5.1 \mathrm{~Hz}, 1 \mathrm{H}), 1.97-1.92(\mathrm{~m}, 1 \mathrm{H}), 1.82-1.70$ $(\mathrm{m}, 1 \mathrm{H}), 1.28-1.23(\mathrm{~m}, 5 \mathrm{H}), 0.94(\mathrm{~s}, 9 \mathrm{H}), 0.92(\mathrm{~s}, 9 \mathrm{H}), 0.86(\mathrm{~s}, 9 \mathrm{H}), 0.11-0.02(\mathrm{~s}, 18 \mathrm{H}) ;{ }^{13} \mathrm{C} \mathrm{NMR}(75 \mathrm{MHz}$, $\left.\mathrm{CDCl}_{3}\right): \delta 171.25,146.68,81.27,78.78,77.58,74.14,73.64,72.69,70.70,64.30,60.49,40.85,30.61,28.80$, $26.70,26.32,26.11,19.10,18.76,18.18,14.18,-3.44,-3.83,-3.90,-4.35,-4.55,-4.74$.

2-((2R,4aS,6S,7R,8S,8aS)-7,8-Bis((tert-butyldimethylsilyl)oxy)-6-((S,E)-1-((tert-butyldimethylsilyl)oxy)-3iodoallyl)octahydropyrano[3,2-b]pyran-2-yl)acetaldehyde (3)<smiles>CCOC(=O)c1ccc([SeH])cc1</smiles>

S17

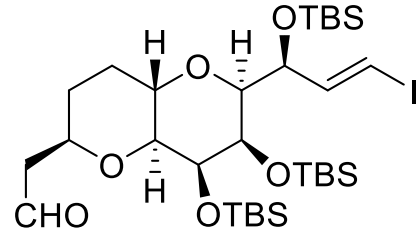

3

To a chilled solution of SI7 $(122.2 \mathrm{~g}, 156 \mathrm{mmol})$ and BHT $(0.75 \mathrm{~g}, 3 \mathrm{mmol})$ in toluene $(1717 \mathrm{~g})$ at $-65^{\circ} \mathrm{C}$ was added DIBAL ( $156 \mathrm{~mL}, 187 \mathrm{mmol}, 1.2 \mathrm{M}$ in toluene) and the reaction mixture was stirred for $1 \mathrm{~h}$. While keeping the reaction temperature below $-55^{\circ} \mathrm{C}$, acetone $(33.8 \mathrm{~mL})$ and toluene $(73 \mathrm{~mL})$ were added. A solution of $\mathrm{MeOH}$ $(19 \mathrm{~mL})$ and toluene $(73 \mathrm{~mL})$ was further added then the reaction mixture was warmed to room temperature. A solution of $1 \mathrm{~N} \mathrm{HCl}(1.3 \mathrm{~L})$ and $\mathrm{MTBE}(818 \mathrm{~mL})$ was added and the reaction mixture was stirred for $10 \mathrm{~min}$. Layers were than separated and organic layer was collected. The organic layer was washed with $1 \mathrm{~N} \mathrm{HCl}(1.3 \mathrm{~L})$, water $(1.2 \mathrm{~L})$, and a solution of $\mathrm{NaHCO}_{3}(1.17 \mathrm{~L}, 5 \%$, aq.). The organic layer was concentrated in vacuo, then to the residue was added heptane $(1.2 \mathrm{~L})$ then mixture was again concentrated in vacuo. The residue was purified by flash chromatography $(\mathrm{Hex} / \mathrm{EtOAc}=10: 1)$ to afford $3(106.5 \mathrm{~g}, 92 \%) .{ }^{1} \mathrm{H} \mathrm{NMR}\left(300 \mathrm{MHz}, \mathrm{CDCl}_{3}\right): \delta 9.79-9.78$ $(\mathrm{m}, 1 \mathrm{H}), 6.85(\mathrm{dd}, J=14.6,7.9 \mathrm{~Hz}, 1 \mathrm{H}), 6.29(\mathrm{dd}, J=14.6,0.7 \mathrm{~Hz}, 1 \mathrm{H}), 4.92-4.88(\mathrm{~m}, 1 \mathrm{H}), 4.11-4.08(\mathrm{~m}, 1 \mathrm{H})$, 3.93-3.81 (m, 3H), 3.53-3.45 (m, 1H), 2.95 (dd, $J=9.6,2.3 \mathrm{~Hz}, 1 \mathrm{H}), 2.63$ (ddd, $J=16.5,8.5,2.6 \mathrm{~Hz}, 1 \mathrm{H}$ ), 2.44 (ddd, $J=16.4,4.4,1.7 \mathrm{~Hz}, 1 \mathrm{H}), 1.98-1.95(\mathrm{~m}, 1 \mathrm{H}), 1.79-1.74(\mathrm{~m}, 1 \mathrm{H}), 1.46-1.32(\mathrm{~m}, 2 \mathrm{H}), 0.94(\mathrm{~s}, 9 \mathrm{H}), 0.93$ (s, 9H), 0.86 (s, 9H), 0.11-0.02 (s, 18H); ${ }^{13} \mathrm{C}$ NMR (75 MHz, $\mathrm{CDCl}_{3}$ ): $\delta 200.78,146.65,81.26,78.97,77.67,73.62$, $72.80,72.65,70.71,64.15,30.88,28.82,26.72,26.28,26.12,19.14,18.76,18.19,-3.42,-3.81,-3.94,-4.37$, 4.57 . 
Copies of ${ }^{1} \mathrm{H}$ and ${ }^{13} \mathrm{C}$ NMR spectra 


\section{Compound 6}

ERC17
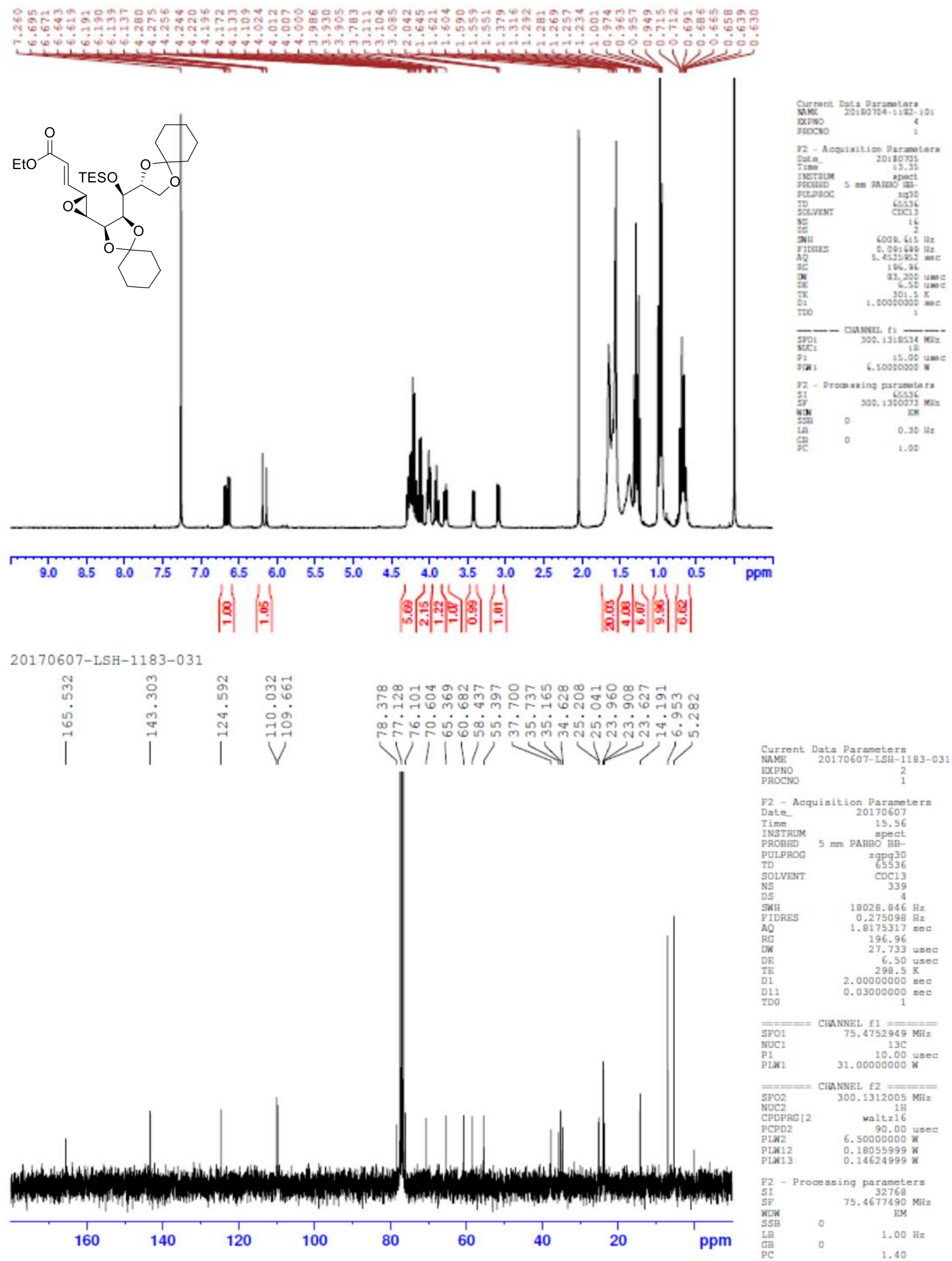

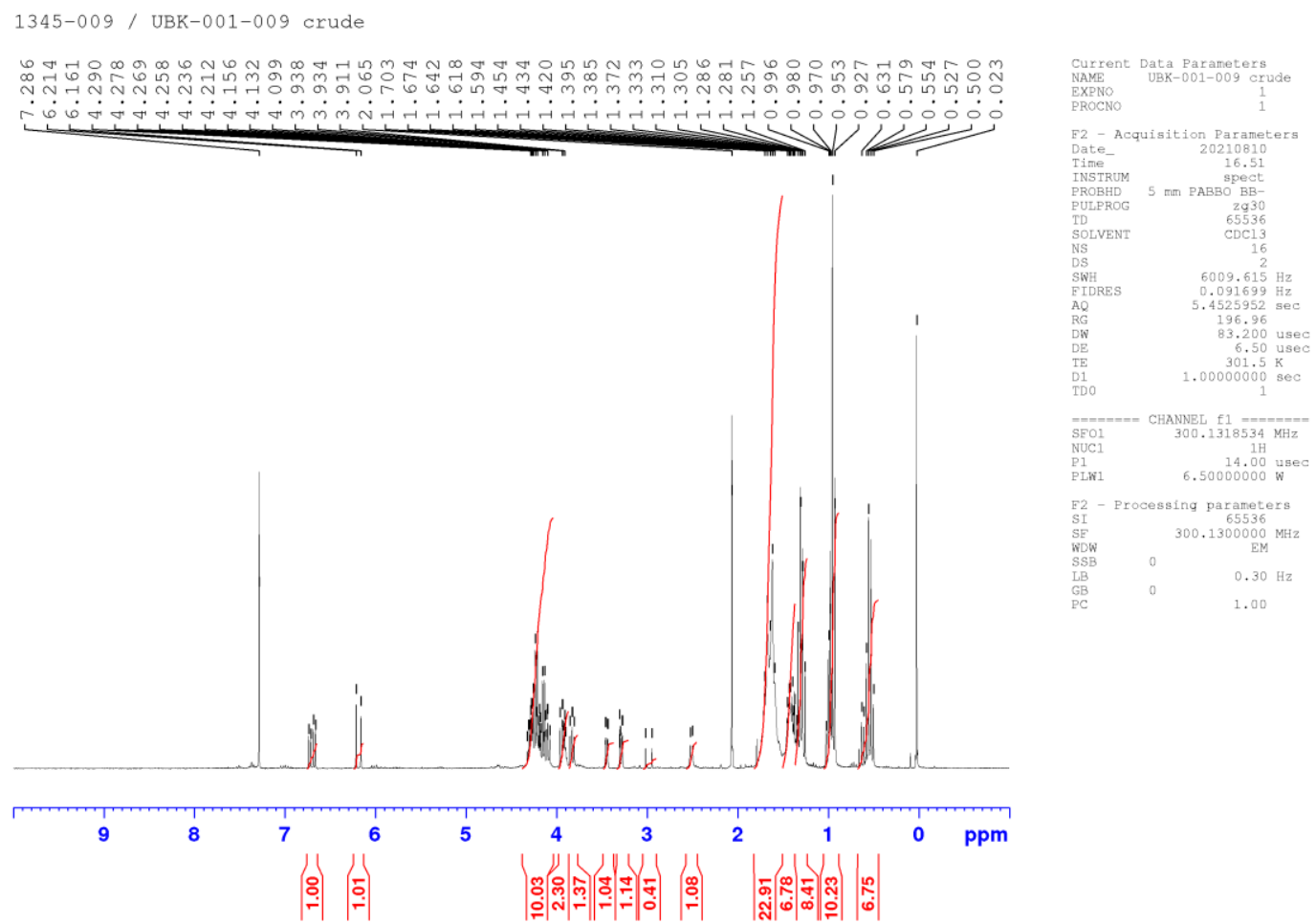


\section{Compound 9}

ERC19
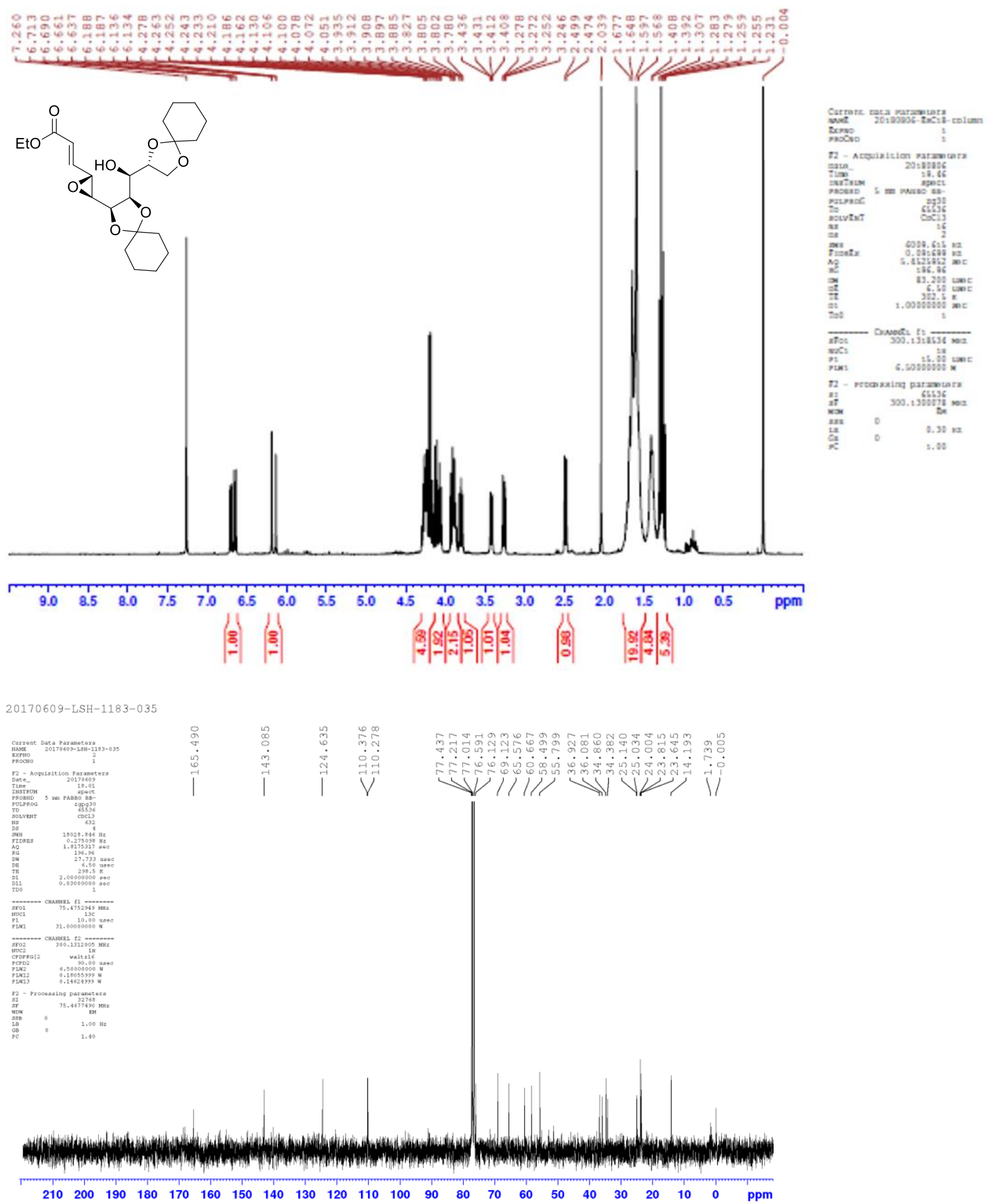


\section{Compound 11}

20171220 ERC16-4

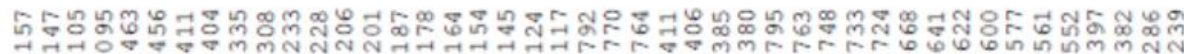

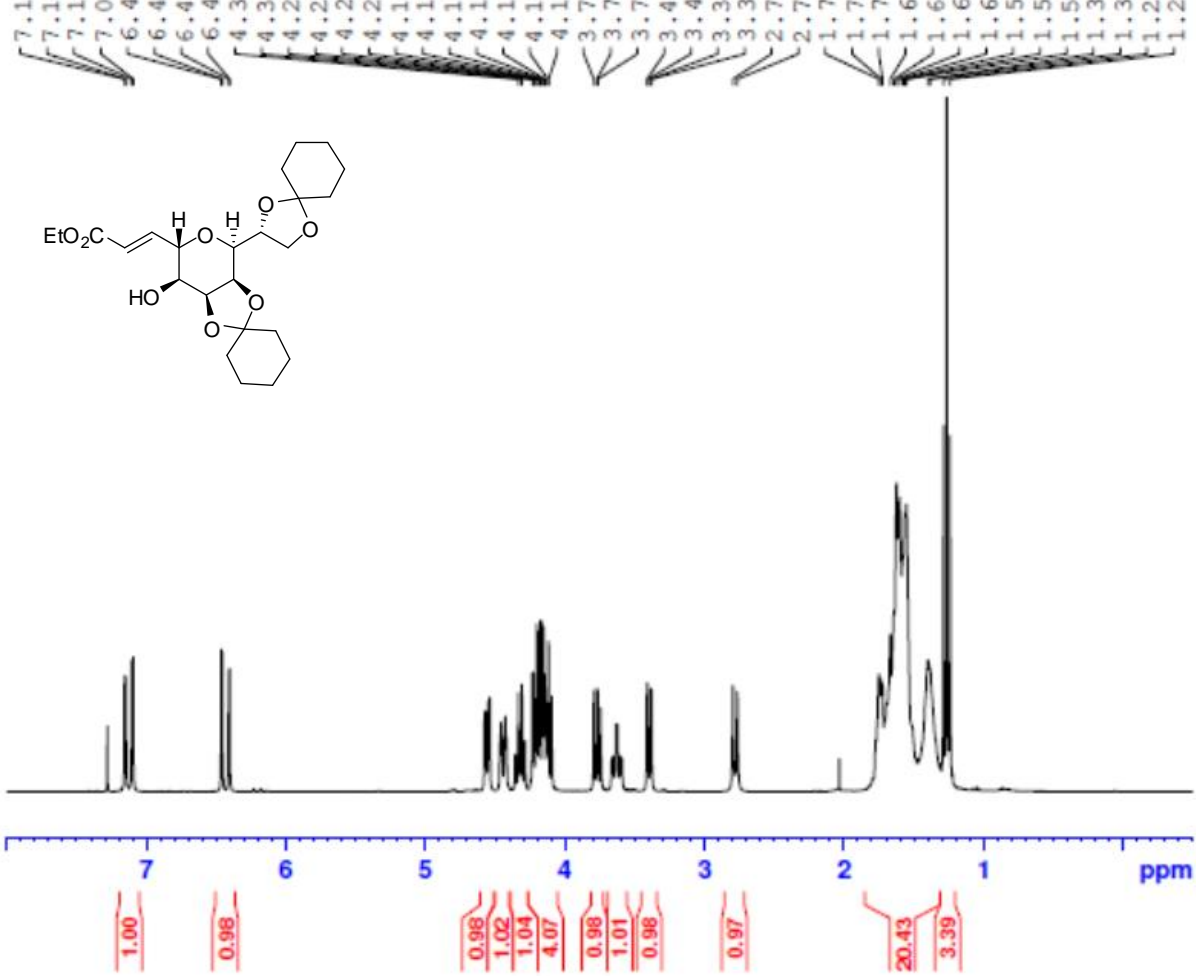

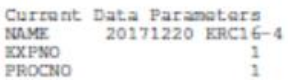

72 - Acquisition Paranaters

Ting 201712.0

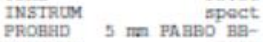

PULPROC $\quad \begin{array}{ll}2430 \\ \text { TD }\end{array}$

sotvest

DS

WH $6009.615 \mathrm{~Hz}$

Ao $\quad 5.6525992 \mathrm{Bz}$

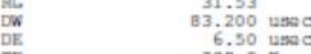

$1.00000000^{293} \mathrm{~K}$

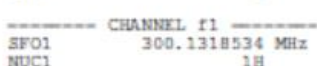

15.00 ugs
P1
PLX1

72 - Procossing paranetara

$\begin{array}{ll}S 1 & 65536 \\ S E & 300.1300000 \\ M H z\end{array}$

$\underset{3 S B}{N D S}$

$\frac{}{\mathrm{CB}}+0.30$

20171220 ERC16-4
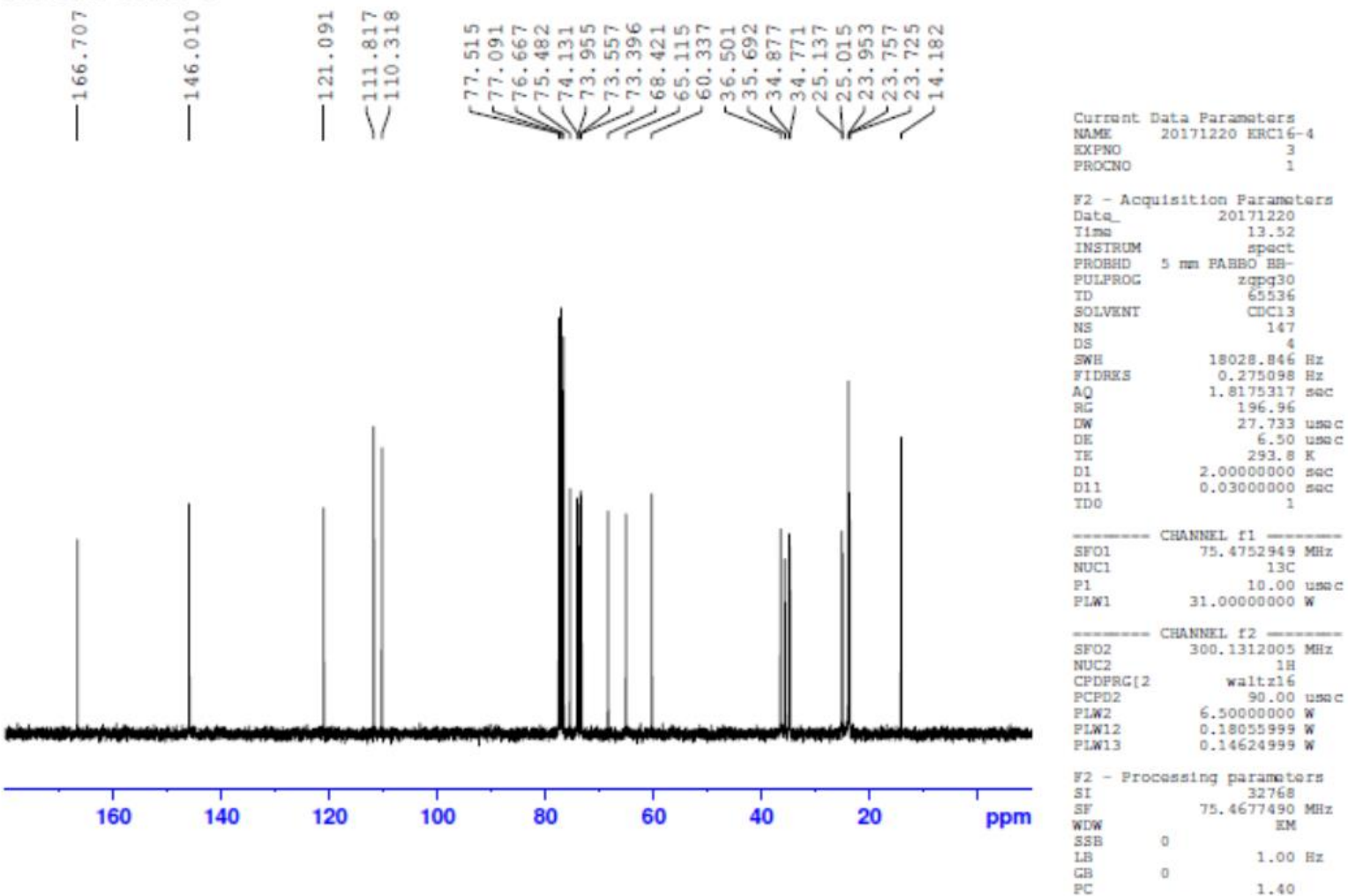

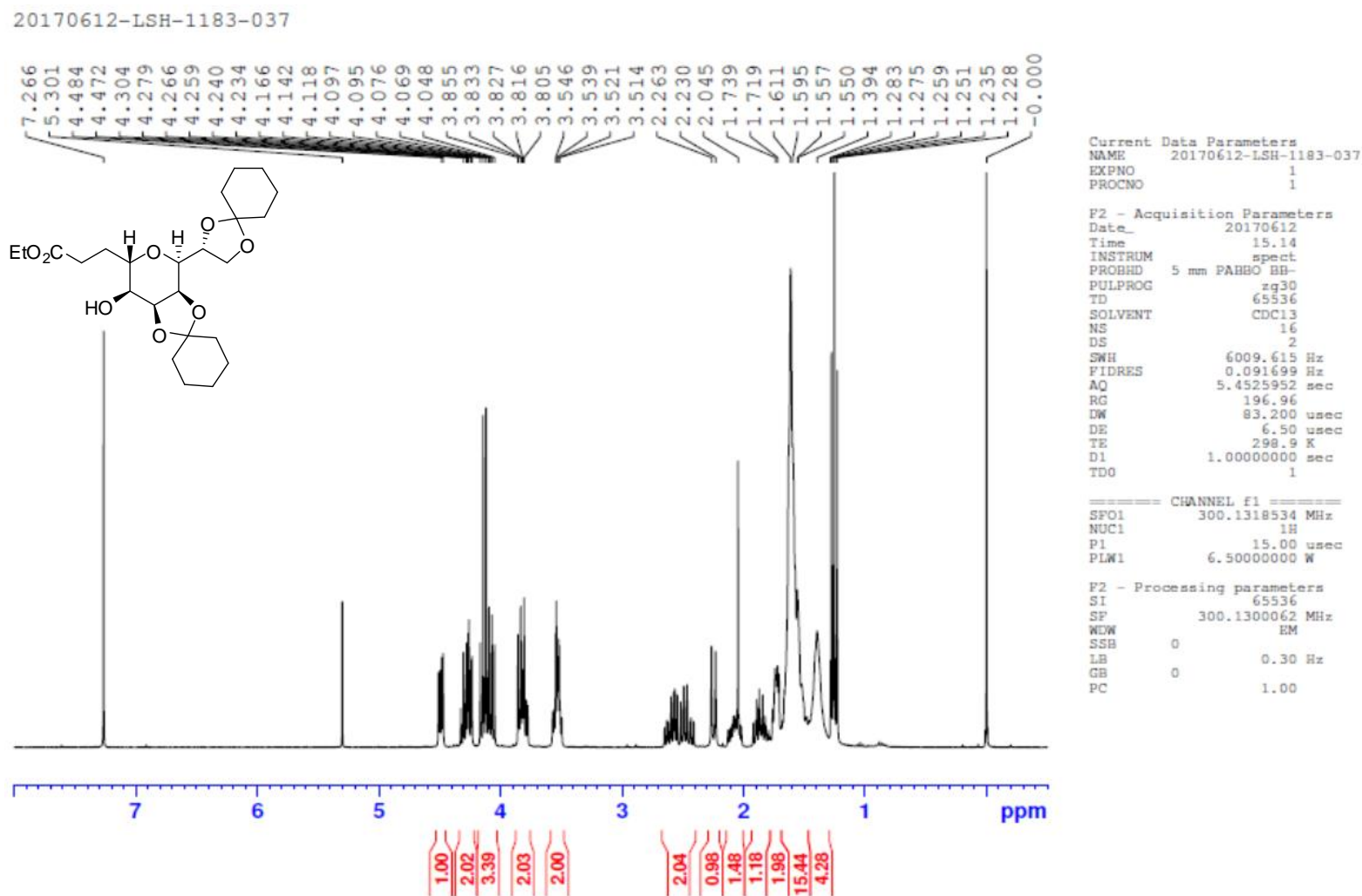

20170612-LSH-1183-037
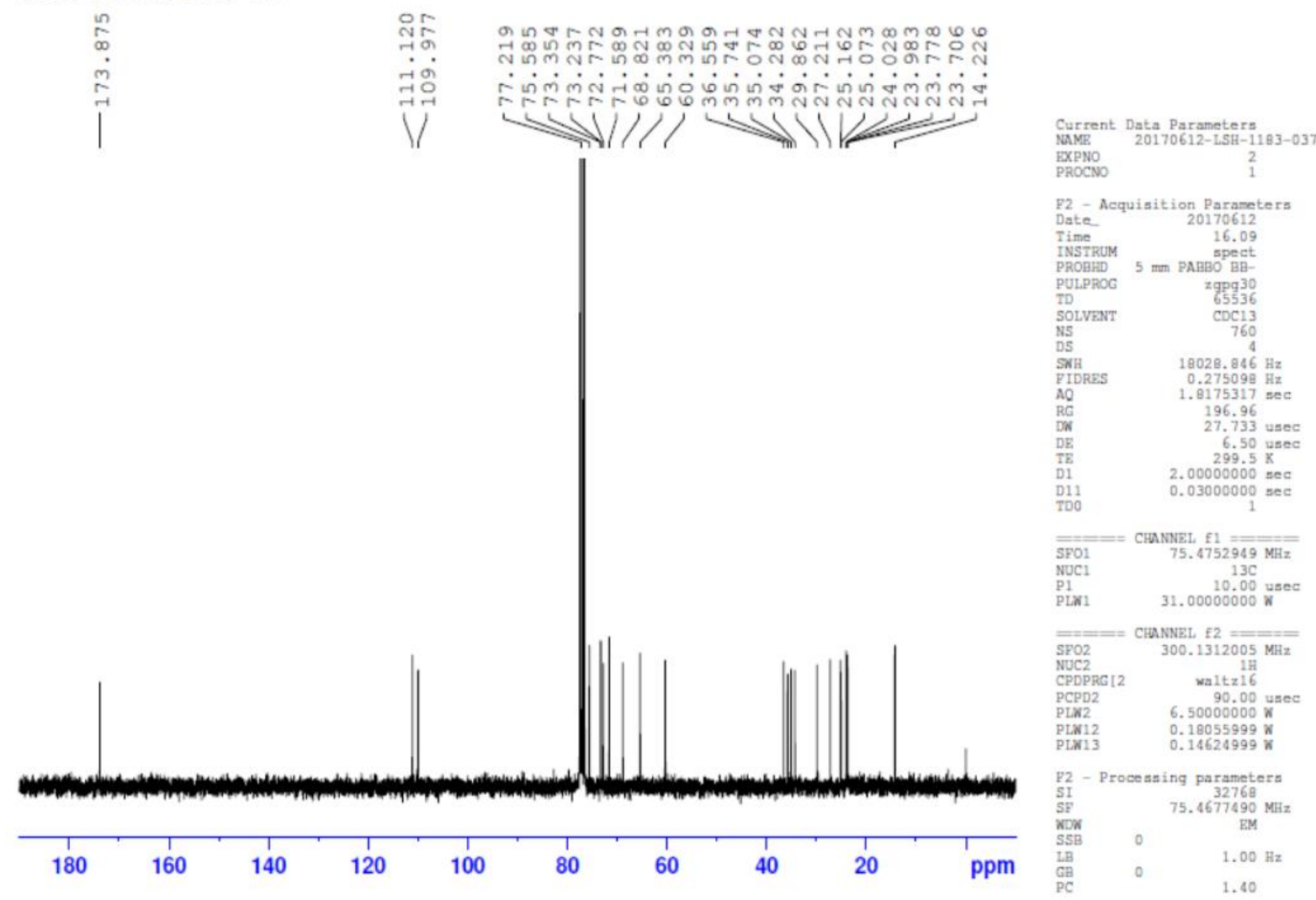


\section{Compound 13}

20160822-LHS-1160-64
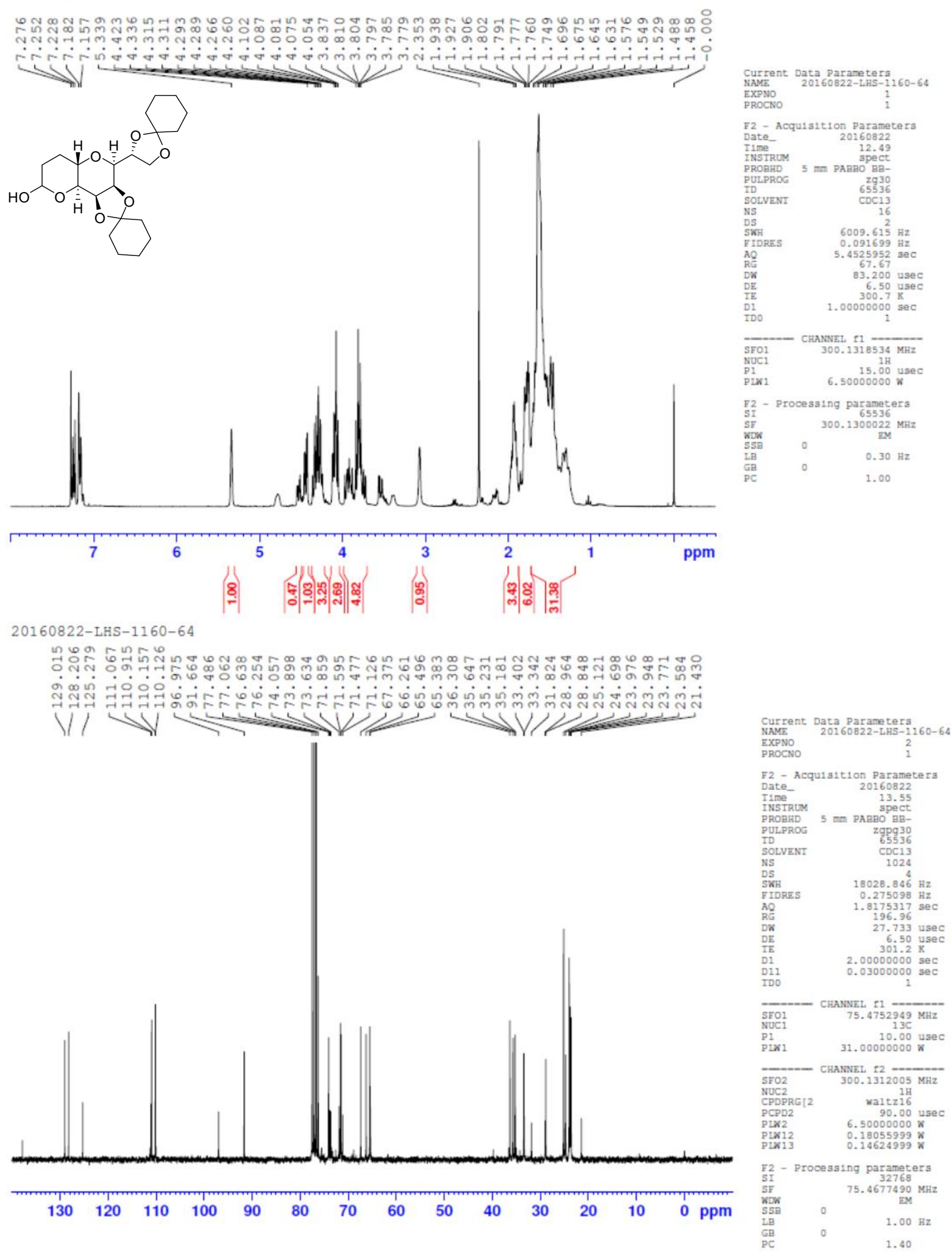

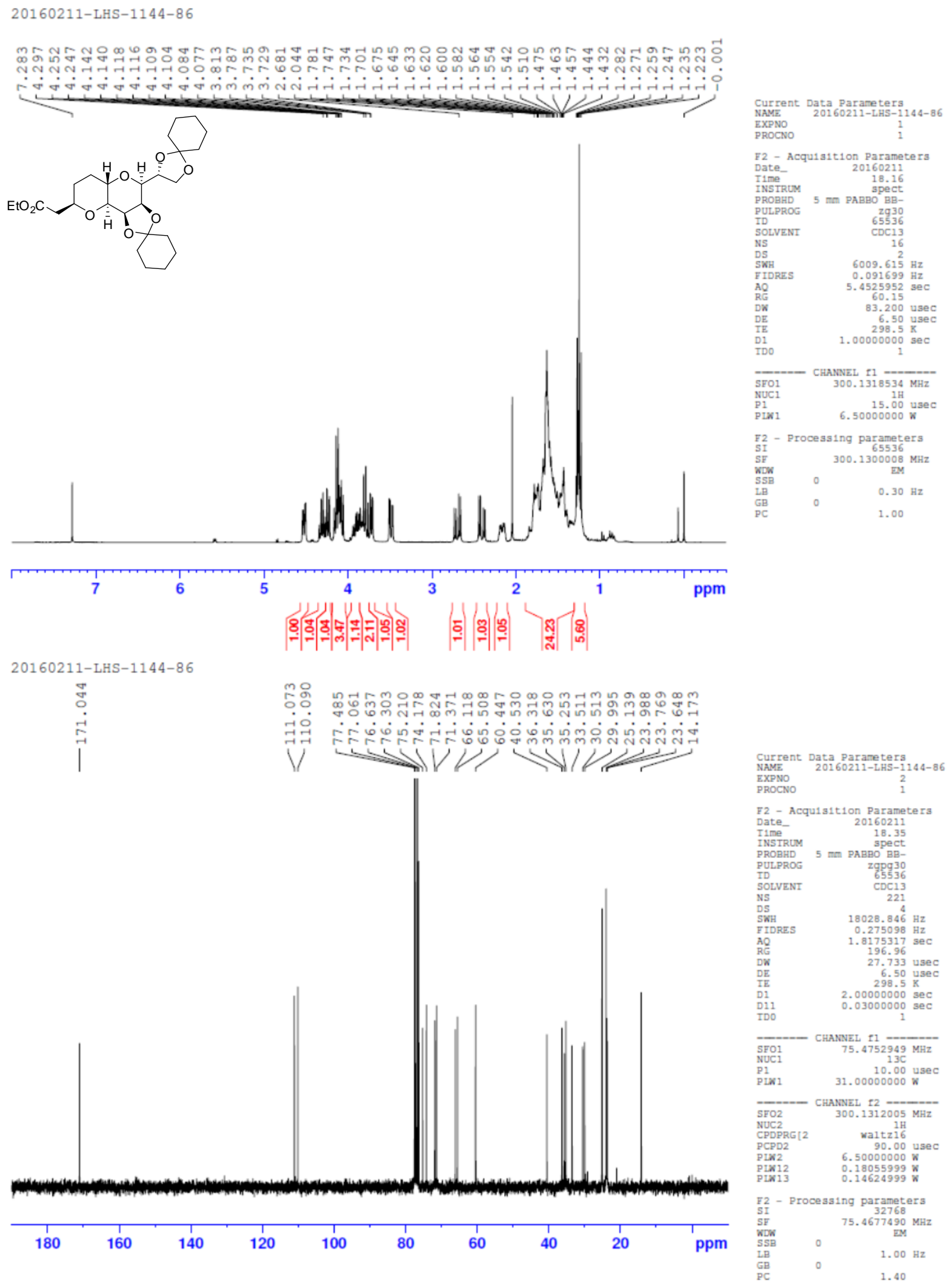


\section{Compound SI2}

20160215-LHS-1144-87

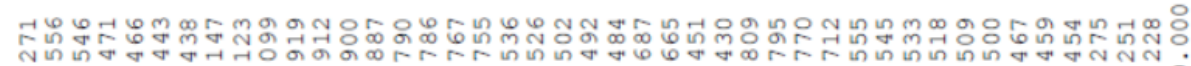

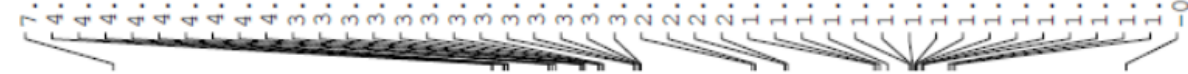<smiles>CCOC(=O)CC1CC[C@H]2O[C@H](C(O)CO)C3OC4(CCCCC4)O[C@H]3[C@H]2O1</smiles>

current Data Paraneters
MME EXPNO
PROCNO

F2 - Acqu1s1tion parameters

T1me- $\quad 16.24$

INSTRCM
PROBHD $5 \mathrm{~mm}$ PABBO BB-

$\begin{array}{lr}\text { PULPROG } & \text { Zq30 } \\ \text { TD } & 65536 \\ \text { SOLVENI } & \text { CDC13 }\end{array}$

$\begin{array}{lr}\text { NS } & 16 \\ \text { DS } & 2 \\ \text { SWH } & 6009.615 \mathrm{~Hz}\end{array}$

$\begin{array}{lr}\text { SWH } & 6009.615 \mathrm{~Hz} \\ \text { FIDRES } & 0.091699 \mathrm{~Hz} \\ \text { AO } & 5.4525952 \mathrm{sec}\end{array}$

$\begin{array}{lr}R G & 113.85 \\ D W & 83.200 \text { usec } \\ \text { DE } & 6.50 \text { use }\end{array}$

$298.5 \mathrm{~K}$
$\mathrm{IE}$

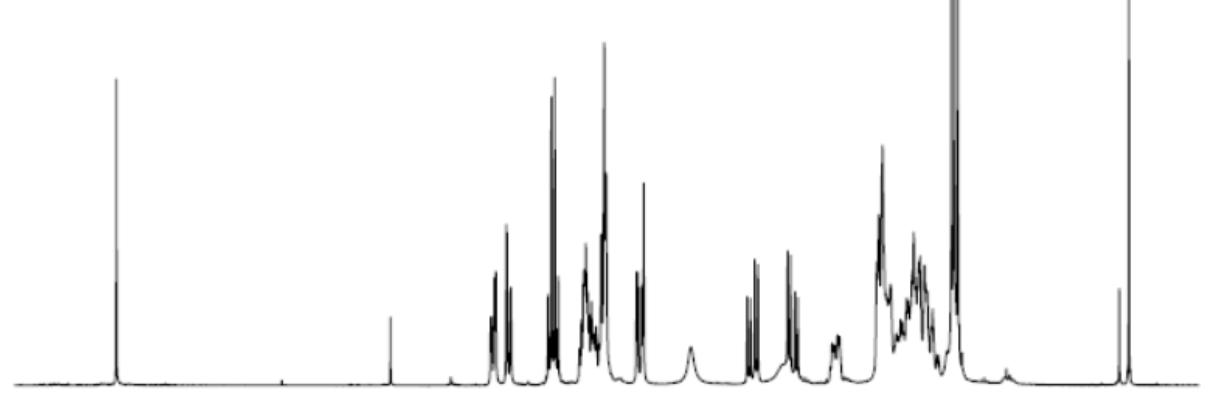

SFO1 CHANNEL $\mathrm{F1}-\mathrm{C}$

15.00 usec

$6.50000000 \mathrm{x}$

F2 - Processing parameters
SI

$\begin{array}{lr}\text { SF } & 300.1300045 \mathrm{MHZ} \\ \text { WDW } & \mathrm{EM}\end{array}$

$\begin{array}{lll}\text { SSB } & 0 & \\ \text { SDB } & 0 & 0.30 \mathrm{HZ} \\ \text { LB } & 0 & \end{array}$

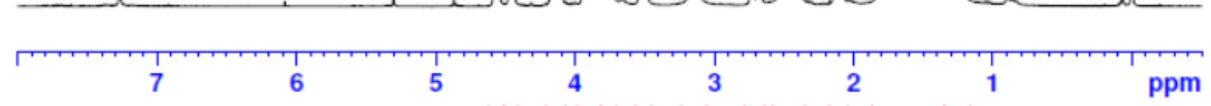

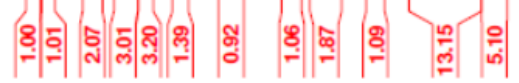

20160215-LHS-1144-87
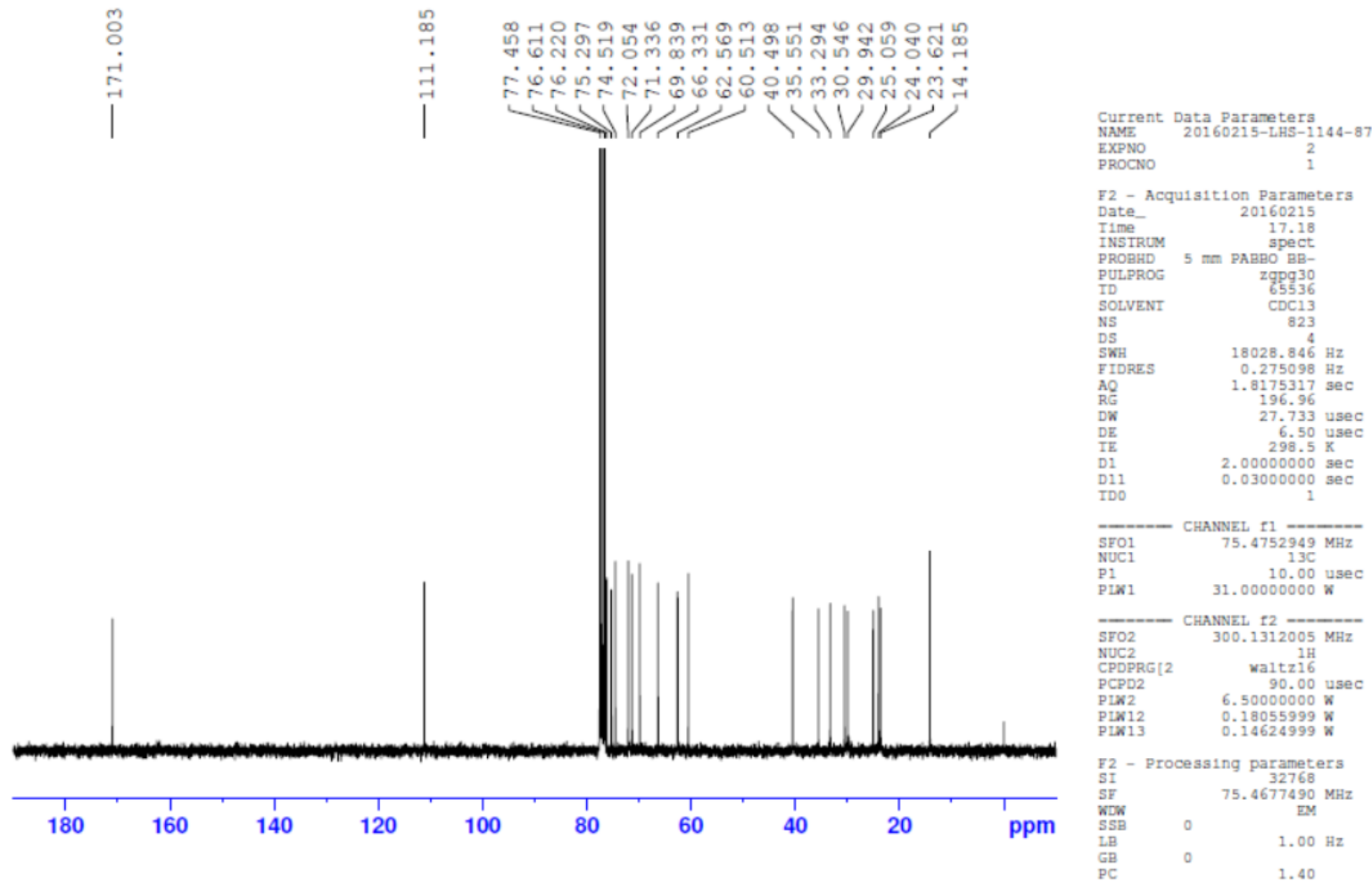
20160217-LHS-1144-90

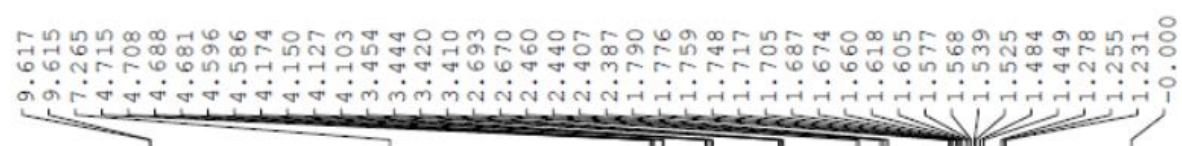

EtO $\mathrm{C}$

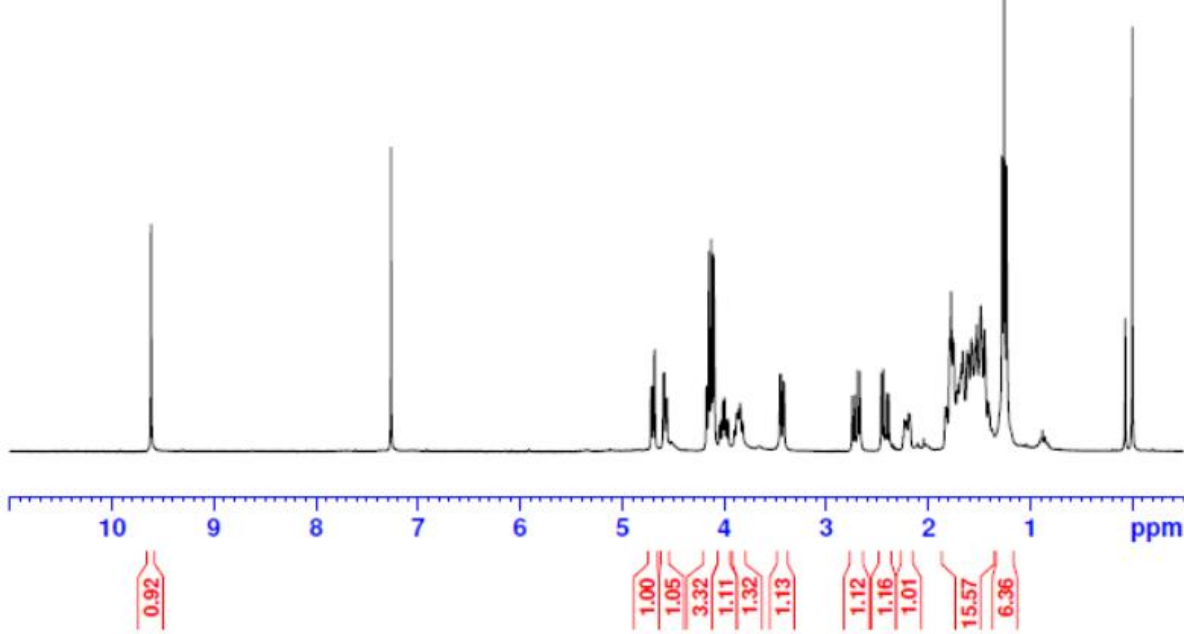

20160217-LHS-1144-90

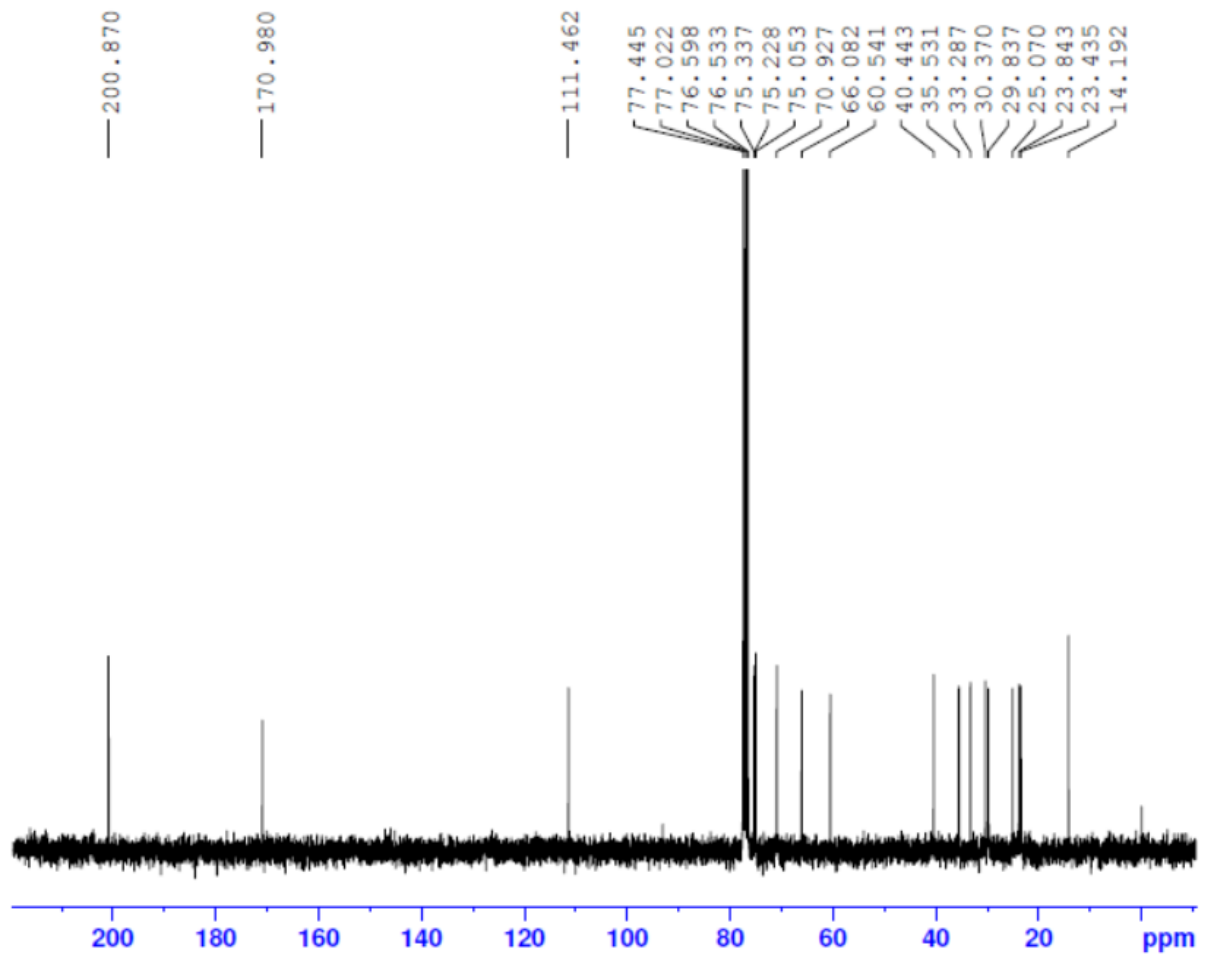

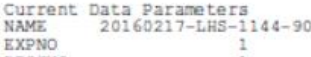

EXPNO

F2 - Acquisition parameters

$\begin{array}{ll}\text { Date_- } & 20160217 \\ \text { Time } & 9.02\end{array}$

spect
INSTREM
PROBD 5 mI PABBO BA-

2930
PULPROG
TD

is

$\begin{array}{ll}\text { DS } & \\ \text { SWH } & 6009.615 \mathrm{~Hz} \\ \text { FIDRES } & 0.091699 \mathrm{~Hz} \\ \text { AO } & 5.4525952 \mathrm{sec} \\ \text { AO } & 157.29\end{array}$

\begin{tabular}{rr}
15 & 83.290 usec \\
\hline$E$ & 6.50 usec
\end{tabular}

$1.00000000 \mathrm{sec}$

SFO1 CHANNEL $\mathrm{In}_{300.1318534 \mathrm{MHz}}$

NUC1 15, $1 \mathrm{H}$ use

$6.50000000 \mathrm{~K}$

F2 - processing parameters

IF $\quad 300.1300064 \mathrm{MHz}$

$\begin{array}{lll}\underset{\mathrm{SBB}}{\mathrm{LB}} & 0 & 0.30 \mathrm{~Hz} \\ \mathrm{FC} & 0 & \end{array}$

Current Data Paraneters
NAME

EXPNO

F2 - Acquis1tion parameters
Date 20160217

T1me $\quad 9.55$

PROBHD $5 \mathrm{~mm}$ PABBO BB-

$\begin{array}{lr}\text { NS } & 709 \\ \text { DS } & 4 \\ \text { SWH } & 18028.846 \mathrm{~Hz}\end{array}$

$\begin{array}{lr}\text { FIDRES } & 18028.846 \mathrm{~Hz} \\ \text { AO } & 0.275098 \mathrm{~Hz} \\ \text { RE } & 1.8175317 \mathrm{se}\end{array}$

$\begin{array}{rr}R G & 196.96 \\ D W & 27.733 \text { usec } \\ D E & 6.500 \text { usec } \\ \text { DE } & 298.5 \mathrm{~K}\end{array}$

$2.00000000 \mathrm{se}$

D11

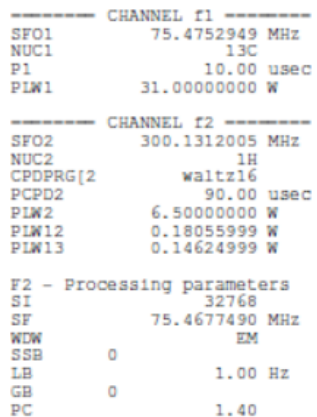




\section{Compound SI4}

ERC4O
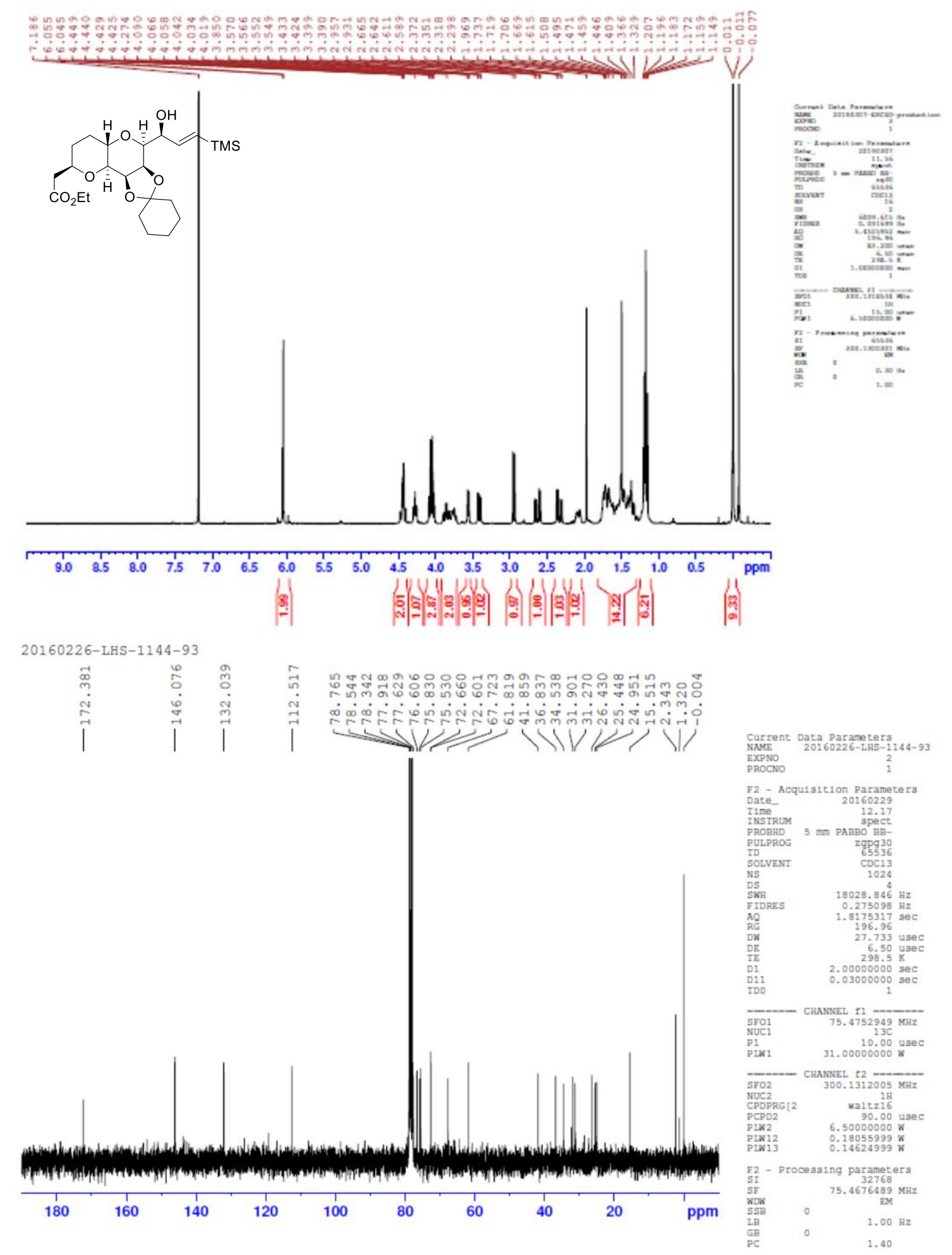


\section{Compound SI5}
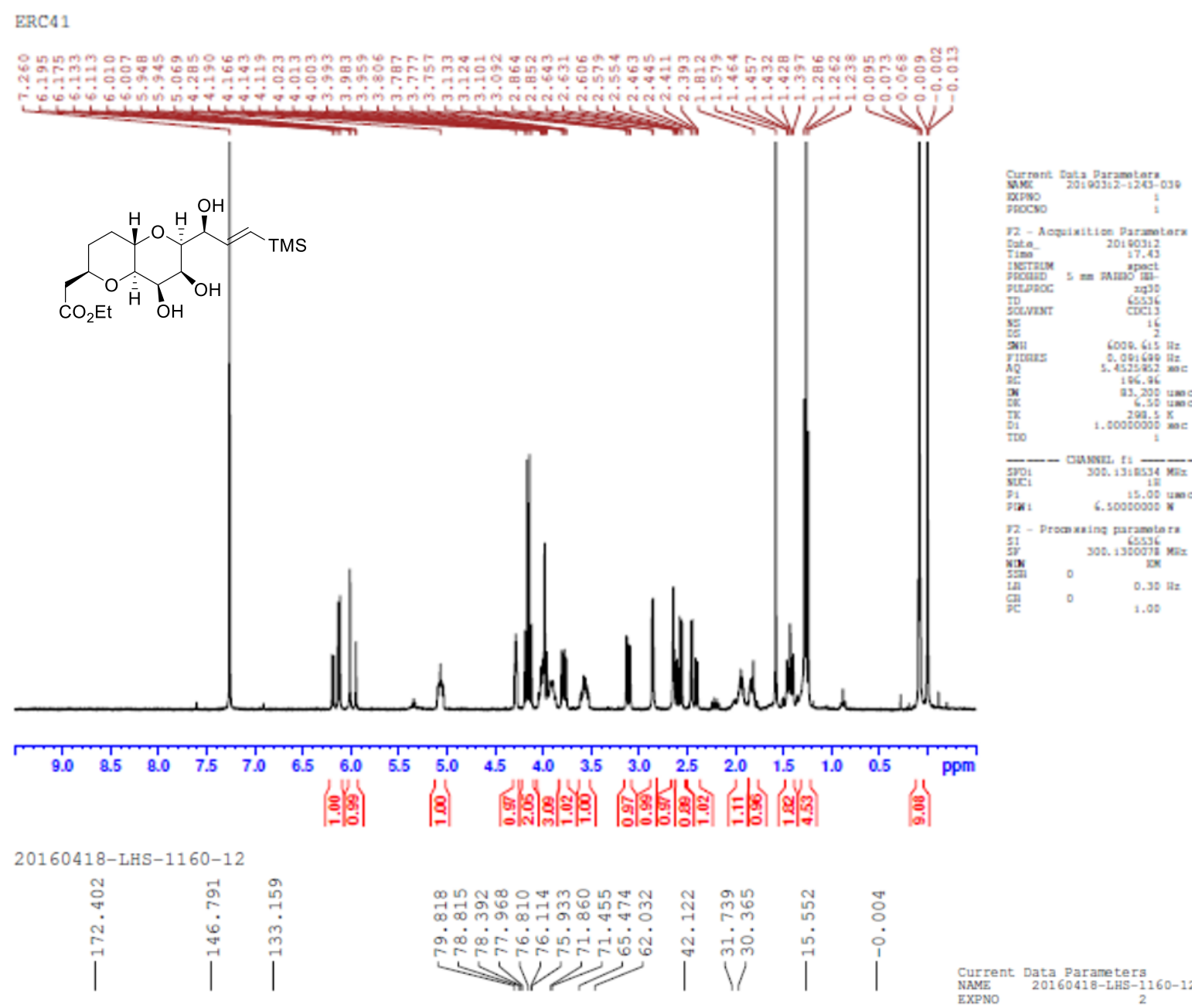

$\begin{array}{lc}\text { Current } & \text { Data Paraneters } \\ \text { NMME } & 20160418-L H S-1160-12 \\ \text { EXPNO } & 2 \\ \text { PROCNO } & 1\end{array}$
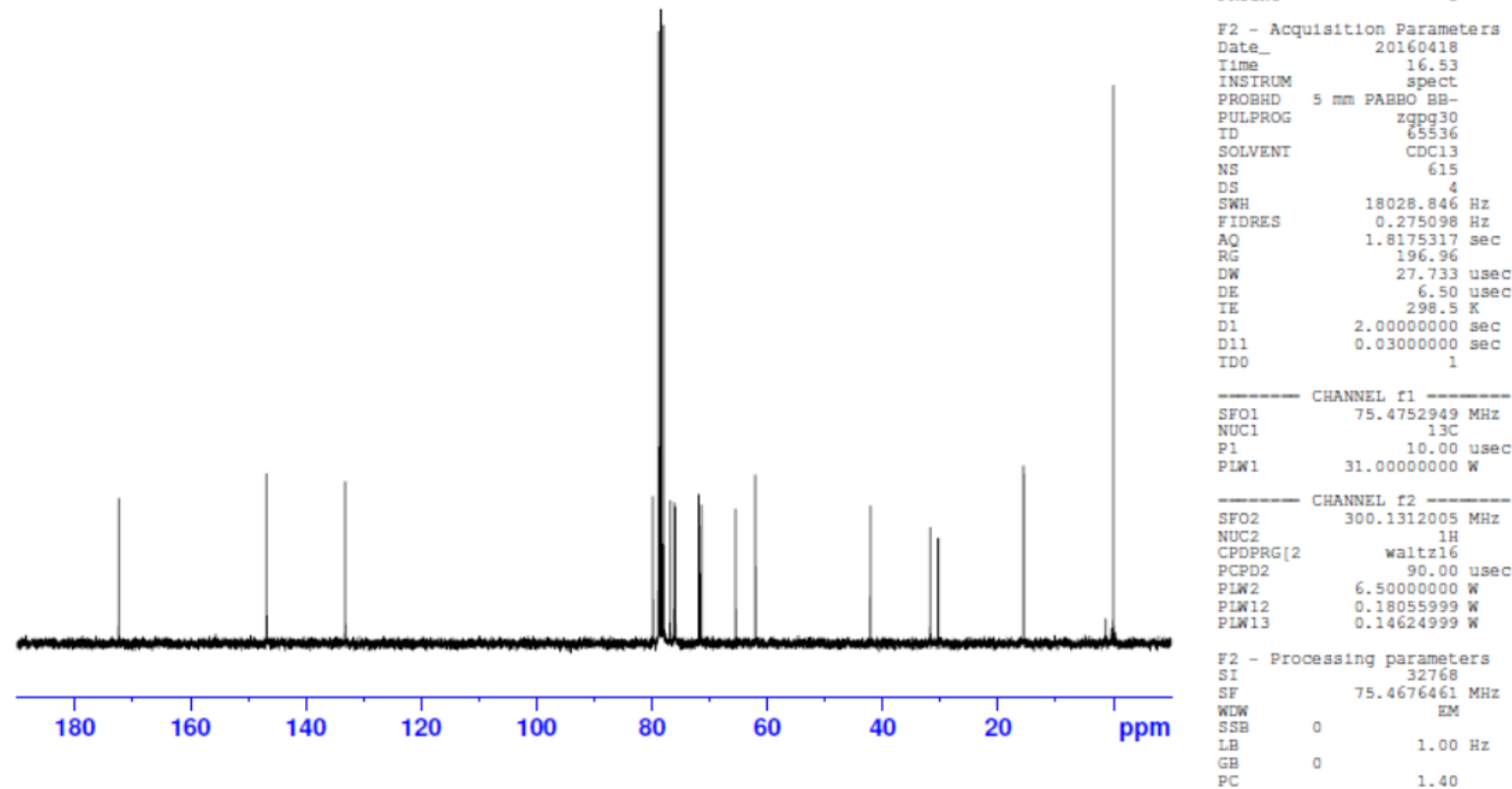


\section{Compound SI6}

ERC42
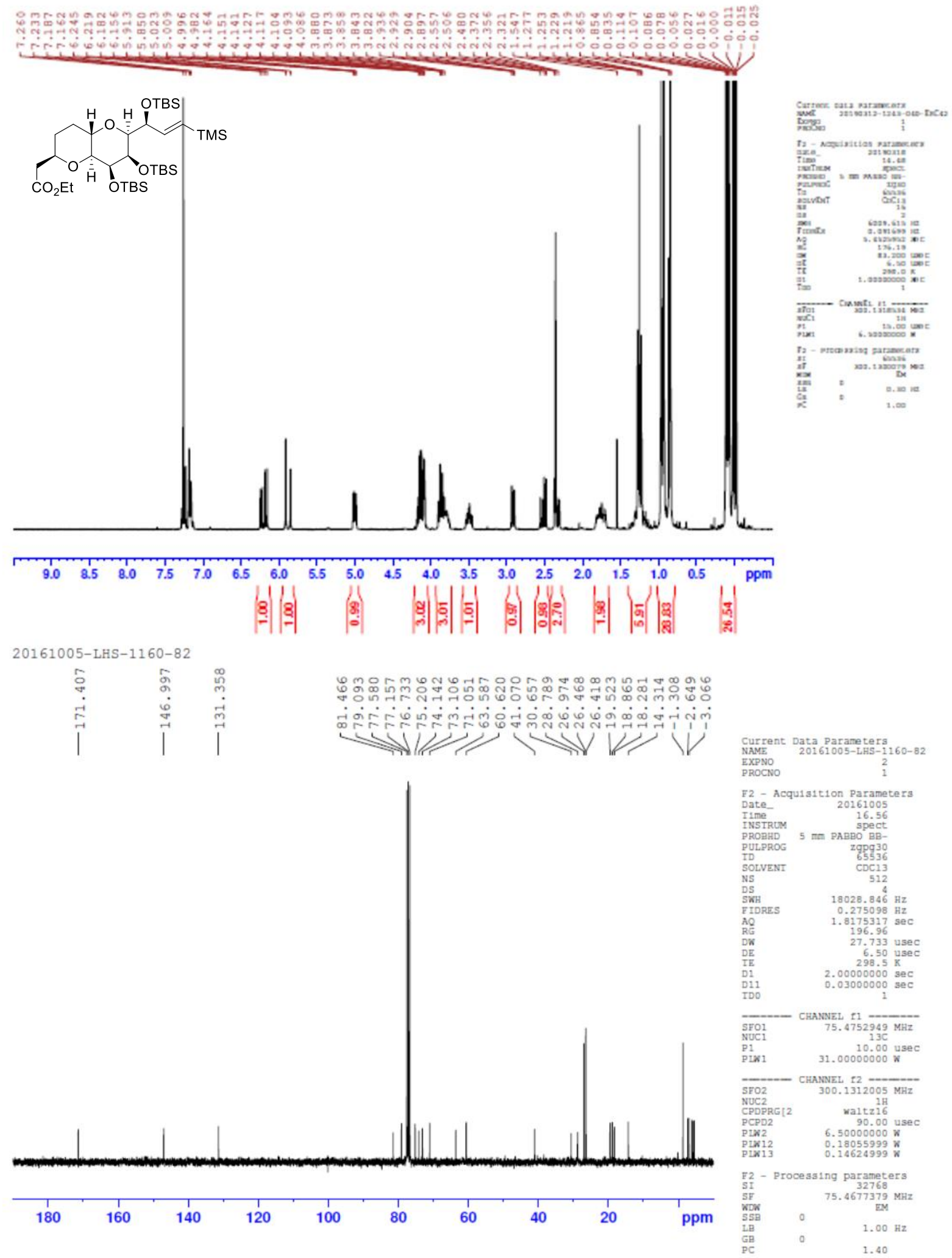
ERC43

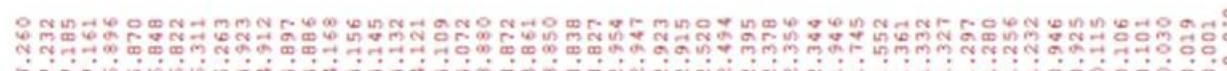

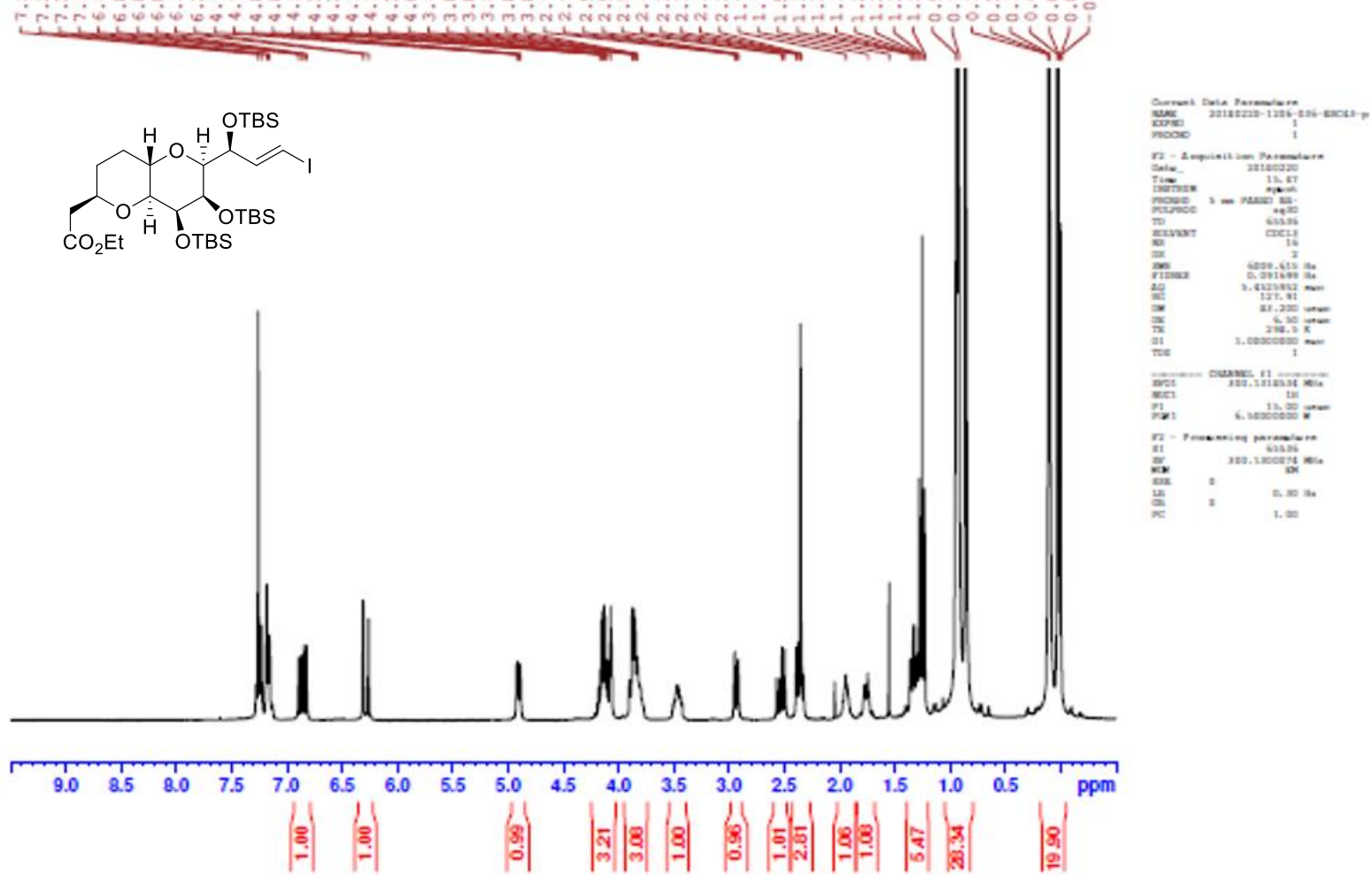

20160423-LHS-1160-19

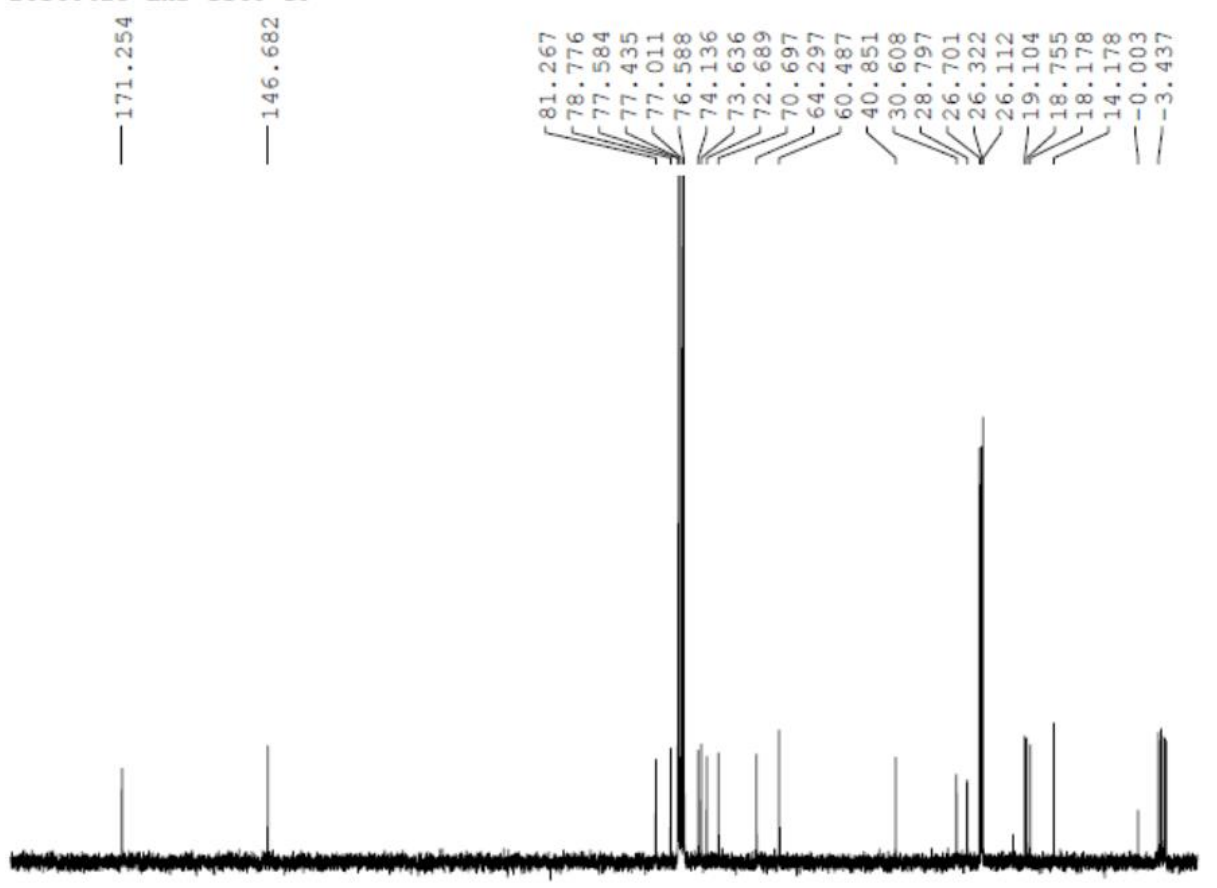

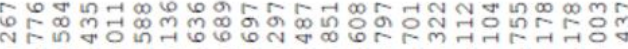

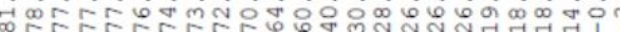

Current Data Paraneters
NAME
EXPNO
20160423-LHS-1160-19 EXPNO

F2 - Acqu131tion Parameters
Date_ $\quad 20160423$
T1me-
P

T1me
INSTRUM
PROPHP

PROAHD $5 \mathrm{~mm}$ PABBO BB-

TD
SOLVENT

NS

$\begin{array}{lr}\text { SWH } & 18028.846 \mathrm{~Hz} \\ \text { FDRES } & 0.275098 \mathrm{~Hz}\end{array}$

$\begin{array}{lr}0.275098 \mathrm{~Hz} \\ \text { AO } & 1.8175317 \mathrm{se}\end{array}$

$\begin{array}{lr}D W & 27.733 \text { usec } \\ \text { DE } & 6.50 \text { usec } \\ \text { TE } & 296.5 \%\end{array}$

D1 $2.00000000 \mathrm{sec}$

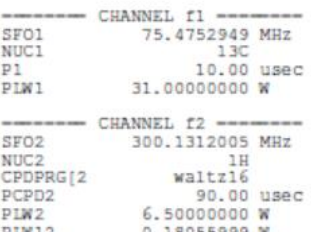

6.50000000

PIW13 0.14624999 К

F2 - Processing parameters
SI
SF
SF
WFE

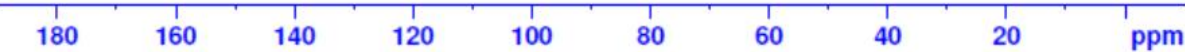




\section{Compound 3}

20170222-LHS-1160-149

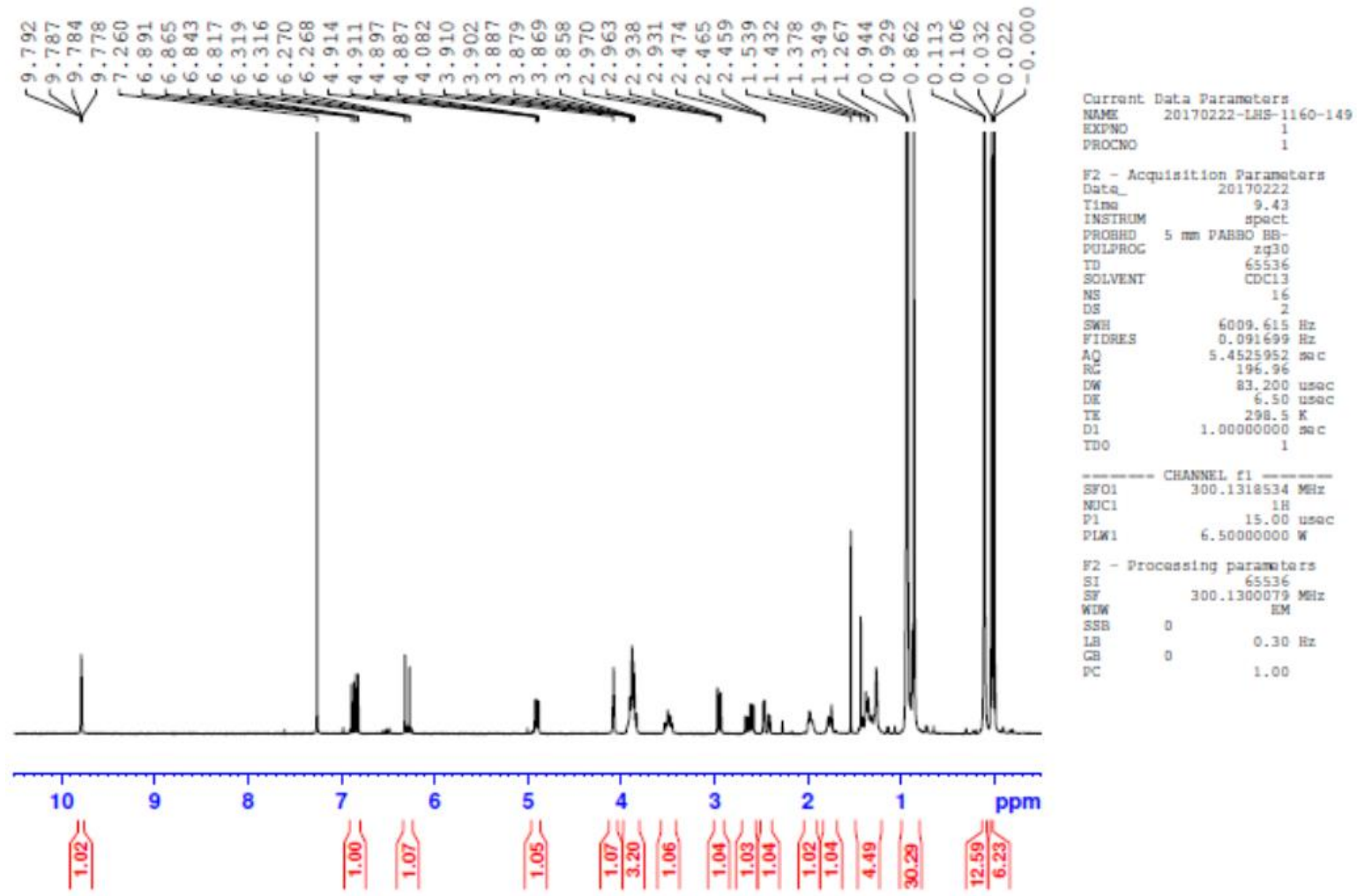

20170222-LHS-1160-149
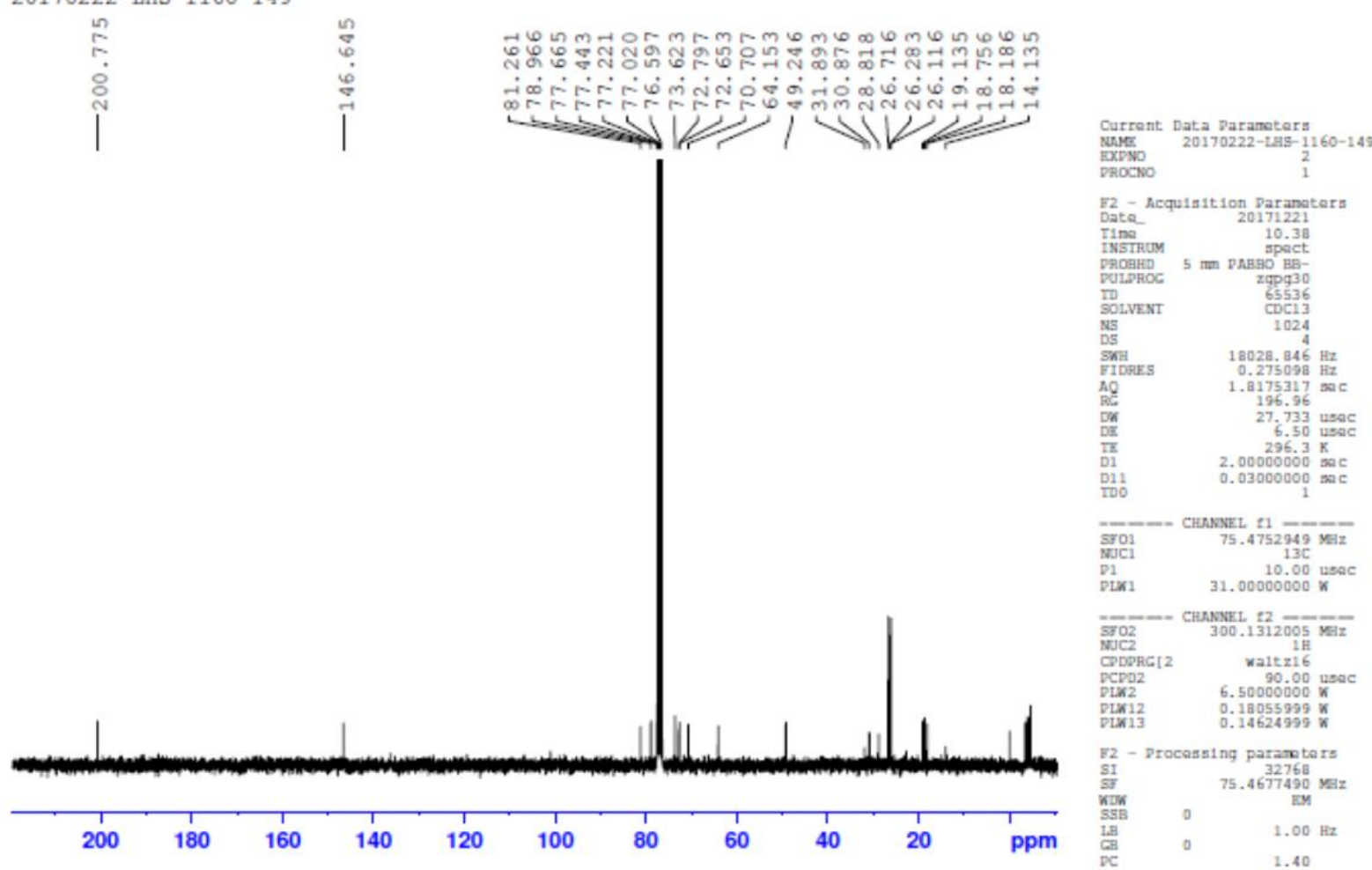